\title{
Magnetic Lieb-Thirring Inequalities
}

\author{
László Erdős ${ }^{\star}$ \\ Department of Mathematics, Princeton University, Princeton, NJ 08544, USA. \\ E-mail: erdlac@math.princeton.edu. Fax: (609)-258-1367
}

Received: 9 May 1994

\begin{abstract}
We study the generalizations of the well-known Lieb-Thirring inequality for the magnetic Schrödinger operator with nonconstant magnetic field. Our main result is the naturally expected magnetic Lieb-Thirring estimate on the moments of the negative eigenvalues for a certain class of magnetic fields (including even some unbounded ones). We develop a localization technique in path space of the stochastic Feynman-Kac representation of the heat kernel which effectively estimates the oscillatory effect due to the magnetic phase factor.
\end{abstract}

\section{Contents}

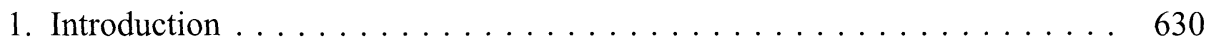

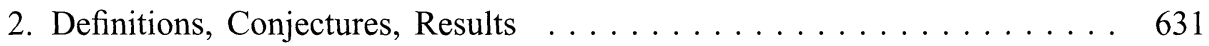

2.1. Magnetic Field with Constant Direction ............. 631

2.2. Magnetic Field with Arbitrary Direction . . . . . . . . . . . . . 635

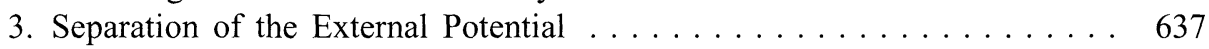

4. Feynman-Kac Formulas . . . . . . . . . . . . . . . . . . . . . . 642

4.1. Two-Dimensional Case . . . . . . . . . . . . . . . . . . 642

4.2. Three-Dimensional Case . . . . . . . . . . . . . . . . . . . . . 644

5. Bounded Magnetic Field with Weak Singularities . . . . . . . . . . . . 647

6. Unbounded Magnetic Field; Reduction to the Main Lemma . . . . . . . . 648

7. Proof of the Main Lemma ... . . . . . . . . . . . . . . 653

7.1. A Sequence of Stopping Times . . . . . . . . . . . . . . 654

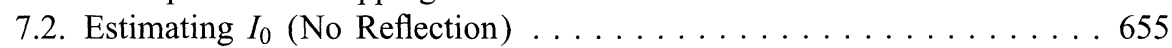

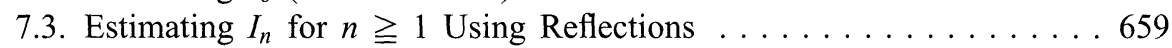

A. Selfadjointness and Negative Essential Spectrum . . . . . . . . . . . 664

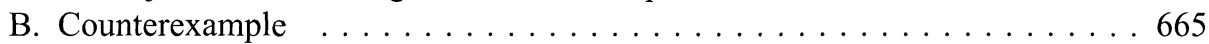

References ............................ 667

^ Work supported by the NSF grant PHY90-19433 A02, by the Alfred Sloan Foundation dissertation Fellowship and by the Erwin Schrödinger Institute for Mathematical Physics in Vienna. 


\section{Introduction}

In this paper, we discuss generalizations of the magnetic Lieb-Thirring inequality obtained in [LSY-II] for the constant magnetic field. The main goal is to obtain reasonable estimates for the moments of the negative eigenvalues of the threedimensional Pauli operator with external potential (describing a nonrelativistic spin$1 / 2$ electron in an electromagnetic field). The basic difference from the previous related works is that we focus on a nonhomogeneous magnetic field.

For the possible applications of this inequality, especially its role played in the proof of the semiclassical formulas, we refer to the papers [LSY-I] and [LSY-II]. Here we just note two requirements that a useful Lieb-Thirring type estimate is expected to fulfill:

- it must be comparable (up to universal constants) with the corresponding semiclassical formula;

- apart from the necessary integrability conditions (which make the semiclassical formula finite) no extra condition should be imposed on the external potential (since in the applications the external potential is usually chosen to be an effective potential whose detailed properties might not be known).

In addition to these basic requirements, we mention that in the related works ([Sob, LSY-I, LSY-II], etc.), special attention is devoted to the case of a strong magnetic field. We also found it physically interesting, and at the same time mathematically difficult, and hence challenging, to treat strong (even unbounded) nonhomogeneous magnetic fields.

There is a vast literature of various spectral studies in the case of the homogeneous magnetic field, but results, especially quantitative ones, are fairly rare for the nonhomogeneous field (see [AHS, CdV, AC, M-1990, M-1991, T]). The technical reason for this (apart from the obvious physical relevance of the constant magnetic field) is twofold. First, the Schrödinger operator with constant magnetic field (without external potential) is exactly solvable, and after decomposing the operator according to the Landau levels one obtains a simplified (lower dimensional) setup, so the additional effect of the external potential becomes easier. Some version of this strategy has almost always been used in any work concerning homogeneous magnetic field.

The second technical difficulty is that perturbations of the magnetic field can be much less controlled than that of the external potential. Naively, one would expect that a local change of the magnetic field does not have a large effect on local quantities observed far away, but the magnetic vector potential, appearing in the operator is a nonlocal quantity (i.e. it undergoes a nonlocal change with a long tail even under local perturbation of the field itself). This is the source of the Aharonov-Bohm effect.

Out basic method is stochastic via the Feynman-Kac formula, which is valid under fairly general conditions on the magnetic field. The analysis of the stochastic oscillatory integral in the Feynman-Kac formula involves a new localization technique in path space which enables us to estimate the heat kernel of the Pauli operator (without external potential). The key idea of this technique has been presented in the simplest possible setup in [E-1994(a)] yielding new pointwise estimates on the magnetic heat kernel. In the present paper we refine this technique to obtain a stronger estimate (unfortunately under more restrictive conditions) which can be 
combined with the Birman-Schwinger principle (in order to include the external potential) to obtain the desired Lieb-Thirring inequality.

\section{Definitions, Conjectures, Results}

The three-dimensional Pauli Hamiltonian is

$$
\mathbf{H}_{\text {Paul } l}:=\mathbf{H}_{\text {Pauli }}^{0}+V \cdot \mathbf{I}:=[(\mathbf{p}-\mathbf{A}) \cdot \boldsymbol{\sigma}]^{2}+V \cdot \mathbf{I},
$$

acting on $L^{2}\left(\mathbf{R}^{3}, \mathbf{C}^{2}\right)$, the Hilbert space of a spin-1/2 particle. Here $\boldsymbol{\sigma}=\left(\sigma_{1}, \sigma_{2}, \sigma_{3}\right)$ stands for the vector of Pauli matrices, $\mathbf{A}$ is the vector potential of the underlying magnetic field $\mathbf{B}(\mathbf{x})=\operatorname{rot} \mathbf{A}(\mathbf{x}), \mathbf{x} \in \mathbf{R}^{3}, \mathbf{I}$ is the $2 \times 2$ identity matrix and $\mathbf{p}=-i \nabla$. Throughout this paper we assume that $\mathbf{A}, \operatorname{div} \mathbf{A}$ and $\mathbf{B}$ are in $L_{l o c}^{2}$.

2.1. Magnetic Field with Constant Direction. In most of our work (except Theorem 2.4), we consider a nonhomogeneous magnetic field with constant direction, i.e. assume that $\mathbf{B}(\mathbf{x})=(0,0, B(\mathbf{x})) \in \mathbf{R}^{3}$. By $\operatorname{div} \mathbf{B}=0$ the function $B(\mathbf{x})$ depends only on the first two coordinates of $\mathbf{x}=\left(x_{1}, x_{2}, x_{3}\right) \in \mathbf{R}^{3}$, which we will denote by $x:=\left(x_{1}, x_{2}\right) \in \mathbf{R}^{2}$. We do not specify the gauge $\mathbf{A}(\mathbf{x})$ here, but we will always restrict ourselves to an appropriate two-dimensional gauge, i.e. $\mathbf{A}(\mathbf{x})=\left(A_{1}(x), A_{2}(x), 0\right)=:(A(x), 0)$ (depending only on $\left.x\right)$. We will use the convention that $\mathbf{A}=\left(A_{1}, A_{2}, A_{3}\right)$ denotes a vectorfield in $\mathbf{R}^{3}$ and $A=\left(A_{1}, A_{2}\right)$ denotes the associated two-dimensional vectorfield, similarly to the convention on the points $\mathbf{x} \in \mathbf{R}^{3}$ and $x \in \mathbf{R}^{2}$.

Under these conditions $\mathbf{H}_{\text {Pauli }}$ decouples into two operators of the form $(\mathbf{p}-\mathbf{A})^{2} \pm B+V$ acting on the spin-up and spin-down subspaces, respectively. If $B(x) \geqq 0$ (as in our main Theorem 2.2), then for the upper bounds on the moments of the negative eigenvalues the difficult part is to study the negative eigenvalues $E_{1} \leqq E_{2} \leqq \cdots \leqq 0$ of

$$
H_{0}:=(\mathbf{p}-\mathbf{A})^{2}-B+V .
$$

The contribution from the eigenvalues $E_{1}^{\prime} \leqq E_{2}^{\prime} \leqq \cdots \leqq 0$ of the other operator $H_{1}:=(\mathbf{p}-\mathbf{A})^{2}+B+V$ can be estimated by the eigenvalue moment of $(\mathbf{p}-\mathbf{A})^{2}+$ $V$. Following the simplest standard proof of the nonmagnetic Lieb-Thirring inequality and applying the diamagnetic inequality, one obtains

$$
\sum_{i}\left|E_{l}^{\prime}\right|^{\gamma} \leqq K \cdot \int_{\mathbf{R}^{3}}|V(\mathbf{x})|_{-}^{\gamma+3 / 2} \mathrm{~d} \mathbf{x} \quad \text { with } \quad K:=\frac{2^{\gamma+1} \gamma}{\pi\left(4 \gamma^{2}-1\right)(2 \gamma+3)}
$$

(see also Remark 4 after Theorem 2.4). Here, and in the sequel, $|V|$ - denotes the negative part of $V$.

Remark. We are not aware of any general theorem that would apriori ensure the selfadjointness of $\mathbf{H}_{\text {Paul }}$ or $H_{0}$ (after imposing some $L^{p}$-bound on $V$ ). The usual theorems about the perturbation of a selfadjoint operator do not seem to work if $B$ is unbounded (which will be our main concern). Nevertheless, the way we will prove our Lieb-Thirring inequalities for unbounded fields implies almost immediately that the operator is semibounded (so it has a self-adjoint extension) and has no negative essential spectrum. The details are found in Appendix A. 
The naive conjecture for the moment of the negative eigenvalues is the following:

Naive conjecture. Assume that $\mathbf{B}$ has constant direction and $B(x) \geqq 0$. Then for any $\gamma>1 / 2$ there exist two absolute constants $C_{1}(\gamma)$ and $C_{2}(\gamma)$ such that for the moments of negative eigenvalues of $H_{0}$ we have

$$
\sum_{i}\left|E_{i}\right|^{\gamma} \leqq C_{1}(\gamma) \int_{\mathbf{R}^{3}} B(x)|V(\mathbf{x})|_{-}^{\gamma+1 / 2} \mathrm{~d} \mathbf{x}+C_{2}(\gamma) \int_{\mathbf{R}^{3}}|V(\mathbf{x})|_{-}^{\gamma+3 / 2} \mathrm{~d} \mathbf{x}
$$

if the integrals above are finite.

Remark. 1. This conjecture is based on the following heuristic argument (see also [LSY-II], where the conjecture was proven for a constant magnetic field). The twodimensional unperturbed operator

$$
H:=\left(p_{1}-A_{1}\right)^{2}+\left(p_{2}-A_{2}\right)^{2}-B=(p-A)^{2}-B
$$

is nonnegative and has a nontrivial zero energy spectral projection $P_{0}$ with a kernel $P_{0}(x, y)$, whose diagonal element $P_{0}(x, x)$ is more or less equal to $B(x) / 2 \pi$. For more precise statements see [E-1993]. Recall that $H_{0}=H+p_{2}^{3}+V$, so over each point $x \in \mathbf{R}^{2}$ the operator $p_{3}^{2}+V$ acting on a one-dimensional fiber gives rise to $\int_{\mathbf{R}}\left|V\left(x, x_{3}\right)\right|_{-}^{\gamma+1 / 2} \mathrm{~d} x_{3}$ as a contribution to the eigenvalue moment. Multiplying it by the density states $\sim B(x) / 2 \pi$ and integrating over $x \in \mathbf{R}^{2}$ one obtains the first term in (4). The second term comes from the contribution of the strictly positive part of the spectrum of $H$ and it has the form as of the usual Lieb-Thirring inequality. The reason for is that $\left(1-P_{0}\right) H$ can be estimated from below by the two-dimensional free Laplacian (in some suitable sense).

Remark. 2. The conjecture above is not true without any further condition on $B$. A counterexample is provided in Appendix B. The spirit of this counterexample suggests a simple but necessary modification in (4), namely $B(x)$ on the righthand side must be replaced by a screened version of $B(x)$ with screening length $\sim B(x)^{-1 / 2}$, i.e. by

$$
\tilde{B}(x):=\left(B * \Phi_{B(x)^{1 / 2}}\right)(x)
$$

where $\Phi \geqq 0$ is a $C^{\infty}$-function supported on the unit disc with $\int \Phi=1$, and $\Phi_{\varepsilon}(x):=\varepsilon^{2} \Phi(\varepsilon x)$.

Our methods are too weak to deal with magnetic fields if there is a substantial difference between $B(x)$ and $\tilde{B}(x)$; more precisely, whenever we are able to prove (4), the conditions will automatically imply that $B(x)$ and $\tilde{B}(x)$ are comparable, uniformly in $x$. Therefore we will concentrate on proving (4). The discussion of a different (much rougher) modification of $B$ is found in [E-1994(b)].

First we present a simple Lieb-Thirring type estimate.

Theorem 2.1. Assume that $\mathbf{B}$ has constant direction, $\mathbf{B}(\mathbf{x})=(0,0, B(x))$ with $B=B^{<}+B^{>}$, where $B^{<} \in L^{\infty}\left(\mathbf{R}^{2}\right), B^{>} \in L^{p}\left(\mathbf{R}^{2}\right)$ (for some $p>1$ ) and $B^{>} \geqq 0$, furthermore $|V|_{-} \in L^{\gamma+1 / 2}\left(\mathbf{R}^{3}\right) \cap L^{\gamma+3 / 2}\left(\mathbf{R}^{3}\right)$ for some $\gamma>1 / 2$ (in case of $p=2$ 
the condition $B>\geqq 0$ can be dropped). For the negative eigenvalues $E_{1, P} \leqq$ $E_{2, P} \leqq \cdots \leqq 0$ of $\mathbf{H}_{\text {Paul }}$, we have

$$
\begin{aligned}
\sum_{l}\left|E_{i, P}\right|^{\gamma} \leqq & K_{1}\left(\left\|B^{<}\right\|_{\infty}+c_{2}(p)\left\|B^{>}\right\|_{p}^{p /(p-1)}\right) \int_{\mathbf{R}^{3}}|V(\mathbf{x})|_{-}^{\gamma+1 / 2} \mathrm{~d} \mathbf{x} \\
& +K_{2} \int_{\mathbf{R}^{3}}|V(\mathbf{x})|_{-}^{\gamma+3 / 2} \mathrm{~d} \mathbf{x}
\end{aligned}
$$

where

$$
K_{1}:=\frac{2^{\gamma+1} \gamma e \cdot k_{2}^{2}(p)}{\pi\left(4 \gamma^{2}-1\right)} \cdot \frac{\mu+1}{1-\lambda}
$$

and

$$
K_{2}:=\frac{3 \cdot 2^{\gamma+3} \gamma e^{2}}{\pi^{3 / 2}\left(4 \gamma^{2}-1\right)(2 \gamma+3)} \cdot \frac{k_{2}^{2}(p)}{\lambda^{2}\left(1-e^{-\mu}\right)^{2}},
$$

with $0<\lambda<1$ and $\mu>0$ being free parameters. The constants $k_{d}(p)$ and $c_{d}(p)(d=2,3)$ depend only on the exponent $p$ and dimension $d$ :

$$
c_{d}(p):=(2 \pi)^{-\frac{d}{2 p-d}}\left(1-\frac{1}{p}\right)^{\frac{d(p-1)}{2 p-d}} \cdot \Gamma\left(1-\frac{d}{2 p}\right)^{\frac{2 p}{2 p-d}}
$$

(where $\Gamma$ denotes the gamma function), while $k_{d}(p)$ can be expressed by the Mittag-Leffler functions (see Remark 3.1 in $[C]$ ). In case of $B^{>}=0$, one can always replace $k_{2}(p)$ by 1 in (8).

Remark. 1. We remark that the above estimate is not consistent dimensionally, unless $B^{>}=0$ (bounded field), since $\left\|B^{<}\right\|_{\infty}$ and $\left\|B^{>}\right\|_{p}^{p /(p-1)}$ scale differently. This discrepancy is especially striking when we take the Planck constant into account (see later). We believe that one should be able to replace the power $p /(p-1)$ by 1 in (7).

Remark. 2. Theorem 2.1 does not impose any further condition $B$ apart from $B \in L^{\infty}+L^{p}$, even $B \geqq 0$ is not assumed. But the estimate is weaker than (4) unless we have positive lower and upper bounds $0<B_{0} \leqq B(x) \leqq C \cdot B_{0}$ (in which case the constants in (4) will depend on $C$ ). For magnetic fields that are close to zero on some domain, (4) would definitely be stronger than (7). At the same time, the counterexample in Appendix B shows that the vanishing magnetic field might cause troubles in the original conjecture. Therefore we will impose a uniform positive lower bound on $B$, and we then address the question of eliminating the condition on the upper bound and obtaining the conjectured form (4) instead of (7). Although the necessary conditions given in Theorem 2.2 below are still very restrictive and the proof of this theorem requires a conceptually new approach, we do obtain the original form, (4), of the naive conjecture by imposing these conditions.

Theorem 2.2. Assume that the magnetic field with constant direction $(\mathbf{B}(\mathbf{x})=$ $(0,0, B(x)))$ has a positive lower bound

$$
0<B_{0} \leqq B(x),
$$


$B(x)$ is continuously differentiable, and for some constant $c$ it satisfies

$$
|B(x)-B(y)| \leqq c \cdot d(x)|x-y|
$$

for any $x, y \in \mathbf{R}^{2}$, where

$$
d(x):=B_{0}^{3 / 2} \cdot\left(\frac{B_{0}}{B(x)}\right)^{31 / 6} .
$$

Then there exist two constants $C_{1}$ and $C_{2}$ depending only on $c$ such that the estimate (4) is valid with

$$
C_{1}(\gamma):=\frac{C_{1} \cdot 2^{\gamma+1} \gamma}{(1-\lambda)\left(4 \gamma^{2}-1\right)} \text { and } C_{2}(\gamma):=\frac{C_{2} \cdot 2^{\gamma+7 / 2} \gamma}{\lambda^{2}\left(4 \gamma^{2}-1\right)(2 \gamma+3)},
$$

where $0<\lambda<1$ is a free parameter.

For example, the conditions (10) and (11) are clearly satisfied with $c=c\left(c^{*}\right)$ if for some $c^{*}$ and for all $x$,

$$
B_{0} \leqq B(x) \leqq c^{*} B_{0}(1+|x|)^{6 / 37} \text { and }|\nabla B(x)| \leqq c^{*} B_{0}^{3 / 2}(1+|x|)^{-1+6 / 37} .
$$

Remark. 1. The condition (10),(11) essentially impose an upper bound on the gradient of $B$. Only a small gradient is allowed in the regions where $B(x)$ is large. Alternatively, the theorem can be formulated in the following way:

Alternative Formulation of Theorem 2.2. Let $c^{\prime}:=B_{0}^{-1 / 2} \cdot \sup _{x}\left(|\nabla b(x)| \cdot|b(x)|^{31 / 6}\right)$, where $b(x):=B(x) / B_{0}$ is the dimensionless magnetic field. Then (4) is valid with constants $C_{1}(\gamma), C_{2}(\gamma)$ which depend on $c^{\prime}$ in a monotone increasing way. Especially, they blow up as $B_{0} \rightarrow 0$.

Remark. 2. The exponent 31/6, which appears in (12) and determines the maximal growth rate of $B$ at infinity (compare with (14)), is necessary for the following proof, but, as we remarked above, the conjecture (4) is expected to hold under much more general circumstances. Therefore this exponent only expresses the limitations of our method and does not have any physical meaning.

Remark. 3. The relations (10),(11) and (12) are almost homogeneous in the magnetic field therefore we have a semiclassical statement as well. If we include the Planck constant in the original Pauli Hamiltonian, $[(h \mathbf{p}-\mathbf{A}) \cdot \boldsymbol{\sigma}]^{2}+V \cdot \mathbf{I}$, then $H_{0}$ becomes

$$
h^{2}\left[\left(\mathbf{p}-\frac{\mathbf{A}}{h}\right)^{2}-\frac{B}{h}+\frac{V}{h^{2}}\right],
$$

so the magnetic field must be rescaled by $h^{-1}$. Notice that this change makes the conditions even weaker (moreover they become irrelevant in the $h \rightarrow 0$ limit). The estimate for the eigenvalue moment is

$$
\sum_{i}\left|E_{i}\right|^{\gamma} \leqq C_{2}(\gamma) \cdot h^{-3} \int|V|_{-}^{\gamma+3 / 2}+C_{1}(\gamma) \cdot h^{-2} \int B|V|_{-}^{\gamma+1 / 2} .
$$

Since $C_{2}(\gamma)$ is not the semiclassical constant, the second term becomes relevant only for large magnetic field $\left(B \geqq h^{-1}\right)$. 
Before going into the details of the proofs, we would like to mention very briefly two other results related to the naive conjecture (4) which can be found in the author's Ph.D. Thesis [E-1994(b)].

One can try to check the naive conjecture directly for exactly solvable models. It turns out that for the "Coulombic" magnetic field, $B(x):=b /|x|$ (with $b>0$ ), the operator (with the natural gauge choice) $H:=(p-A)^{2}-B$ is exactly solvable (and actually it has dense point spectrum in the interval $\left[0, b^{2}\right]$, similar to a phenomenon investigated qualitatively in [MS]). Using explicit formulas for the eigenfunctions, one can estimate the spectral density of $H$ with a precision that is sufficient to prove (4). The proof involves various estimates on the asymptotic behavior of the Laguerre polynomials. The significance of this result is that the Coulombic magnetic field is neither bounded from above nor has a positive lower bound (so Theorem 2.2 does not apply), and it shows that the conjecture can be valid even for magnetic fields with a singularity. (Theorem 2.1 can be applied, but it gives a weaker bound than (4).)

The second, related result deals with the azimuthally symmetric situation.

Proposition 2.3. Assume that $B(x)=B(|x|) \geqq 0$ and $V(\mathbf{x})=V\left(|x|, x_{3}\right)$ (where $\left.|x|:=\sqrt{x_{1}^{2}+x_{2}^{2}}\right)$ and let $a(r):=(1 / r) \int_{0}^{r} B(s) s \mathrm{~d} s$ be the absolute value of the natural radial gauge. Then for any $\gamma>0$ there exists a universal constant $c(\gamma)$ such that

$$
\sum_{l}\left|E_{i}\right|^{\gamma} \leqq c(\gamma) \sum_{n \in \mathbf{Z}} \int_{\mathbf{R}} \int_{0}^{\infty}\left|-B(r)+V\left(r, x_{3}\right)+\left(\frac{n}{r}-a(r)\right)^{2}\right|_{-}^{\gamma+1} r \mathrm{~d} r \mathrm{~d} x_{3} .
$$

The main idea of the proof is that one can investigate the problem separately in each angular momentum sector (so that the magnetic field becomes an effective potential) and apply a modified version of the idea of [L]. This estimate is not comparable directly to the original form of the conjecture, but imposes no condition on the magnetic field apart from the symmetry, so it might be useful in some situations when Theorems 2.1 and 2.2 do not apply.

2.2. Magnetic Field with Arbitrary Direction. Here we give a different LiebThirring type inequality which is valid for any bounded magnetic field, possible with some weak singularities $\mathbf{B}(\mathbf{x}) \in L^{\infty}\left(\mathbf{R}^{3}\right)+L^{2}\left(\mathbf{R}^{3}\right)$ (it is not assumed that the field has constant direction).

Theorem 2.4. For any magnetic field $\mathbf{B}=\mathbf{B}^{<}+\mathbf{B}^{>}$with $\mathbf{B}^{<} \in L^{\infty}\left(\mathbf{R}^{3}\right), \mathbf{B}^{>} \in$ $L^{2}\left(\mathbf{R}^{3}\right)$ and for any external potential $V$ with $|V|_{-} \in L^{\gamma}\left(\mathbf{R}^{3}\right) \cap L^{\gamma+3 / 2}\left(\mathbf{R}^{3}\right)$ (for $\gamma \geqq 1)$, the negative eigenvalues $E_{1, P} \leqq E_{2, P} \leqq \cdots \leqq 0$ of $\mathbf{H}_{\text {Paul }}$ satisfy

$$
\begin{aligned}
\sum_{i}\left|E_{i, P}\right|^{\gamma} \leqq & K_{1, P}\left(2\left\|\mathbf{B}^{<}\right\|_{\infty}+9 c_{3}(2)\left\|\mathbf{B}^{>}\right\|_{2}^{4}\right)^{3 / 2} \int_{\mathbf{R}^{3}}|V(\mathbf{x})|_{-}^{\gamma} \mathrm{d} \mathbf{x} \\
& +K_{2, P} \int_{\mathbf{R}^{3}}|V|_{-}^{\gamma+3 / 2} \mathrm{~d} \mathbf{x}
\end{aligned}
$$

with

$$
K_{1, P}:=\frac{4^{\gamma+1} \gamma e \cdot k_{3}^{2}(2)}{\pi^{3 / 2}} \cdot(1+\mu)^{3 / 2}
$$


and

$$
K_{2, P}:=\frac{3 \cdot 2^{\gamma+7} \gamma e^{2}}{\pi^{3 / 2}\left(4 \gamma^{2}-1\right)\left(2 \gamma^{+3}\right)} \cdot \frac{k_{3}^{2}(2)}{\left(1-e^{-\mu}\right)^{2}},
$$

where $\mu>0$ is a free parameter, and $c_{3}(2), k_{3}(2)$ are defined in Theorem 2.1 ( for $\mathbf{B}^{>}=0, k_{3}(2)$ can be replaced by 1$)$.

Remark. 1. This inequality is essentially different from the naive conjecture (4) (exponents are different), and, naturally, the heuristic argument given there is not valid for a magnetic field with variable direction. One might naively think that assuming $|V|_{-} \in L^{\gamma}\left(\mathbf{R}^{3}\right) \cap L^{\gamma+3 / 2}\left(\mathbf{R}^{3}\right)$, the estimate (18) is better than (7) for small $\left\|B^{<}\right\|_{\infty}$ (and assuming for the moment that $B^{>}=0$ ), although the conditions are weaker. The point is that the constant in front of the $\int|V|_{-}^{\gamma+3 / 2}$ term is bigger in (18), more precisely by a simple Hölder's inequality

$$
\begin{aligned}
& K_{1}\left\|B^{<}\right\|_{\infty} \int|V|_{-}^{\gamma+1 / 2}+\left(K+K_{2}\right) \int|V|_{-}^{\gamma+3 / 2} \\
& \quad \leqq \frac{2}{3} K_{1}^{3 / 2}\left\|B^{<}\right\|_{\infty}^{3 / 2} \int|V|_{-}^{\gamma}+\left(\frac{1}{3}+K+K_{2}\right) \int|V|_{-}^{\gamma+3 / 2},
\end{aligned}
$$

justifying that (7) together with (3) is, in fact, a stronger estimate than (18) (by appropriately tuning the free parameters in $K_{1}$ and $K_{2}$ one can always achieve that $2 K_{1}^{3 / 2} / 3 \leqq 2^{3 / 2} K_{1, P}$ and $\left.1 / 3+K+K_{2} \leqq K_{2, P}\right)$.

Remark. 2. Let us introduce the Planck constant into $\mathbf{H}_{\text {Paul }}$ and into the estimate (18) (assuming again that $B^{>}=0$, since that term is not correct dimensionally and causes a blow up of order $h^{-6}$ in the semiclassical statement). One obtains that after the main term $\left(h^{-3} \int|V|_{-}^{\gamma+3 / 2}\right)$ the next term is $h^{-3 / 2}\left\|\mathbf{B}^{<}\right\|_{\infty}^{3 / 2} \int|V|_{-}^{\gamma}$. This does not mean any ambiguity about the $h$-power of the second term when compared with (16). One should again keep in mind that the constant in front of the main term is not optimal; the second term in both estimates (7) and (18) becomes relevant only if $h\left\|\mathbf{B}^{<}\right\|_{\infty}$ is at least of order 1 .

Remark. 3. The general conjecture for any (possible unbounded and/or variable direction) magnetic field, analogous to (4), would be that under some minor condition on $\mathbf{B}$,

$$
\sum_{i}\left|E_{i, P}\right|^{\gamma} \leqq C_{1, P}(\gamma) \int_{\mathbf{R}^{3}}|\mathbf{B}(\mathbf{x})||V(\mathbf{x})|_{-}^{\gamma+1 / 2} \mathrm{~d} \mathbf{x}+C_{2, P}(\gamma) \int_{\mathbf{R}^{3}}|V(\mathbf{x})|_{-}^{\gamma+3 / 2} \mathrm{~d} \mathbf{x}
$$

for $\gamma>1 / 2$. This estimate would be consistent with the conjectured semiclassical formula. Theorem 2.4 is much weaker than (21). There is a possible intermediate estimate.

$$
\sum_{i}\left|E_{i, P}\right|^{\gamma} \leqq C_{1, P}^{*}(\gamma) \int_{\mathbf{R}^{3}}|\mathbf{B}(\mathbf{x})|^{3 / 2}|V(\mathbf{x})|_{-}^{\gamma} \mathrm{d} \mathbf{x}+C_{2, P}^{*}(\gamma) \int_{\mathbf{R}^{3}}|V(\mathbf{x})|_{-}^{\gamma+3 / 2} \mathrm{~d} \mathbf{x}
$$

for $\gamma \geqq 1$. It is clear by Hölder's inequality, that (21) is stronger than (22) (not bothering about the constants). On the other hand, the relation between Theorem 2.4 and (22) is clearly the same as the relation between Theorem 2.1 and Theorem 2.2. One can try to apply the ideas of the proof of Theorem 2.2 to prove (22) 
(under some conditions similar to those in Theorem 2.2). The stochastic reflection method, leading to Main Lemma 6.2 in Sect. 7, still works in higher (especially in three) dimensions, see [E-1994(a)]. For the three-dimensional Pauli operator one needs a generalized Feynman-Kac formula which includes a Poisson process as well (see Proposition 4.2), but the reflections can be done and the necessary estimates can be obtained uniformly for each realization of the Poisson process. There is no spectral gap (in the case of a field with constant direction it is implied by the positive lower bound on the magnetic field via supersymmetry-see Sect. 6), but one can still cut the spectrum into two parts at a level $L=B_{0}:=\inf _{\mathbf{x}}|\mathbf{B}(\mathbf{x})|$ (see Sect. 3). Unfortunately, this takes into account all the low lying states of $\mathbf{H}_{\text {Pault }}^{0}$ with an equal weight, although they do not contribute equally to the negative eigenvalues (higher energy states are expected to contribute less). This overestimate explains why we obtain the rougher estimate (22) instead of (21). There are some other technical difficulties, especially about the validity of the FeynmanKac formula for the Pauli operator with a truly unbounded magnetic field (not just weak singularities), but these are probably tractable. The point is that without the spectral gap (e.g. for variable direction field) our heat kernel method is very likely able to prove only (22), but not (21). We do not see any way for the moment to overcome the absence of supersymmetry in the general three-dimensional case.

Remark. 4. We briefly remark that if the so-called electron $g$ factor is smaller than 2 (see [FLL]), then one can easily reduce the magnetic Lieb-Thirring inequality to the standard non-magnetic one (see $[\mathrm{LT}]$ ). In this case one considers $\mathbf{H}_{g}:=$ $(\mathbf{p}-\mathbf{A})^{2}-\frac{g}{2} \boldsymbol{\sigma} \cdot \mathbf{B}$ instead of $\mathbf{H}_{\text {Paul }}^{0}$, and clearly

$$
\mathbf{H}_{g}=\frac{g}{2}\left((\mathbf{p}-\mathbf{A})^{2}-\boldsymbol{\sigma} \cdot \mathbf{B}\right)+\left(1-\frac{g}{2}\right)(\mathbf{p}-\mathbf{A})^{2} \geqq\left(1-\frac{g}{2}\right)(\mathbf{p}-\mathbf{A})^{2}
$$

Plugging this estimate into the full Birman-Schwinger kernel $\mathbf{K}_{E}$ (see (46) later), taking the trace of its square and applying the diamagnetic inequality we can follow the standard proof of the usual (non-magnetic) Lieb-Thirring inequality. The result is $($ for $\gamma>1 / 2)$

$$
\sum_{l}\left|E_{l, P}\right|^{\prime} \leqq \frac{2^{\gamma+2} \gamma}{\pi\left(4 \gamma^{2}-1\right)(2 \gamma+3)}\left(\frac{2}{2-g}\right)^{3 / 2} \int_{\mathbf{R}^{3}}|V(\mathbf{x})|_{-}^{\gamma+3 / 2} \mathrm{~d} \mathbf{x}
$$

for the moments of the negative eigenvalues of $\mathbf{H}_{g}+V$.

\section{Separation of the External Potential}

For the proof of Theorems 2.1 and 2.2, we follow the method of [LSY-II]. The key idea is to split the Birman-Schwinger kernel (recall that $\left.H=(p-A)^{2}-B\right)$

$$
K_{E}:=\left|V+\frac{E}{2}\right|_{-}^{1 / 2}\left(H+p_{3}^{2}+\frac{E}{2}\right)^{-1}\left|V+\frac{E}{2}\right|_{-}^{1 / 2}
$$


$(E>0)$ into a lower and an upper part at level $L, K_{E}=K_{E, L}^{<}+K_{E, L}^{>}$, defined as

$$
\begin{gathered}
K_{E, L}^{<}:=\left|V+\frac{E}{2}\right|_{-}^{1 / 2} \Pi_{L}\left(H+p_{3}^{2}+\frac{E}{2}\right)^{-1} \Pi_{L}\left|V+\frac{E}{2}\right|_{-}^{1 / 2}, \\
K_{E, L}^{>}:=\left|V+\frac{E}{2}\right|_{-}^{1 / 2}\left(1-\Pi_{L}\right)\left(H+p_{3}^{2}+\frac{E}{2}\right)^{-1}\left(1-\Pi_{L}\right)\left|V+\frac{E}{2}\right|_{-}^{1 / 2},
\end{gathered}
$$

where we let $P_{L}$ be the spectral projection onto $[0, L]$ in the spectrum of $H$ (which is nonnegative), and let $\Pi_{L}:=P_{L} \otimes$ Id be its natural extension to $L^{2}\left(\mathbf{R}^{3}\right)$. (According to the heuristic argument outlined in the previous section we should choose $L=0$, but sometimes the splitting is technically more convenient at a positive $L$.) Using Lemma 2.3 in [LSY-II] (about the compactness of these operators see Appendix A) we have

$$
N_{E} \leqq(1-\lambda)^{-1} \operatorname{Tr}\left(K_{E}^{<}\right)+\lambda^{-2} \operatorname{Tr}\left[\left(K_{E}^{>}\right)^{2}\right],
$$

for $N_{E}$, the number of eigenvalues of $H_{0}:=H+p_{3}^{2}+V$ less than $-E(0<\lambda<1$ is a free parameter, $E>0$ ). Naturally

$$
\begin{aligned}
\sum_{l}\left|E_{l}\right|^{\gamma}= & \gamma \int_{0}^{\infty} N_{E} E^{\gamma-1} \mathrm{~d} E \leqq \gamma(1-\lambda)^{-1} \int_{0}^{\infty} \operatorname{Tr}\left(K_{E, L}^{<}\right) E^{\gamma-1} \mathrm{~d} E \\
& +\gamma \lambda^{-2} \int_{0}^{\infty} \operatorname{Tr}\left[\left(K_{E, L}^{>}\right)^{2}\right] E^{\gamma-1} \mathrm{~d} E
\end{aligned}
$$

Using that

$$
P_{L} \leqq e^{t L} \cdot e^{-t H}
$$

(for any $t \geqq 0$ ), a simple calculation, similar to (2.15) in [LSY-II], shows that

$$
\begin{aligned}
\operatorname{Tr}\left(K_{E, L}^{<}\right) & \leqq \operatorname{Tr}\left[\left|V+\frac{E}{2}\right|_{-}^{1 / 2} e^{t L} e^{-t H}\left(p_{3}^{2}+\frac{E}{2}\right)^{-1}\left|V+\frac{E}{2}\right|_{-}^{1 / 2}\right] \\
& =\frac{1}{\sqrt{2 E}} \int_{\mathbf{R}^{3}}\left|V(\mathbf{x})+\frac{E}{2}\right|_{-} e^{t L} e^{-t H}(x, x) \mathrm{d} \mathbf{x},
\end{aligned}
$$

where the diagonal element of the heat kernel is defined for almost all $x$ as follows:

$$
e^{-t H}(x, x):=\int_{\mathbf{R}^{2}}\left|e^{-t H / 2}(x, y)\right|^{2} \mathrm{~d} y,
$$

and $e^{-t H / 2}(x, y)$ denotes the heat kernel of $H$ (its existence will be discussed in Proposition 4.1). Here we have used that

$$
\operatorname{Tr}(U A U)=\iint U^{2}(x)\left|A^{1 / 2}(x, y)\right|^{2} \mathrm{~d} y \mathrm{~d} x
$$

if $A \geqq 0, A^{1 / 2}$ has a kernel and $U$ is a multiplication operator. The point is that calculating the Hilbert-Schmidt norm of $U A^{1 / 2}$ instead of the trace norm of $U A U$ 
allows us to pass to the integral of the kernels without worrying about the existence of the diagonal element $A(x, x)$. If one defines

$$
A(x)=A(x, x):=\int A^{1 / 2}(x, y) A^{1 / 2}(y, x) \mathrm{d} y=\int\left|A^{1 / 2}(x, y)\right|^{2} \mathrm{~d} y,
$$

then $\operatorname{Tr}(U A U)=\int U^{2}(x) A(x) \mathrm{d} x$ is straightforward. This idea will be used several times in the sequel.

Then, still following the technique of [LSY-II], we obtain

$$
\int_{0}^{\infty} \operatorname{Tr}\left(K_{E, L}^{<}\right) E^{\gamma-1} \mathrm{~d} E \leqq \frac{2^{\gamma+1}}{4 \gamma^{2}-1} \int_{\mathbf{R}^{3}}|V(\mathbf{x})|_{-}^{\gamma+1 / 2} e^{t L} \int_{\mathbf{R}^{2}}\left|e^{-t H / 2}(x, y)\right|^{2} \mathrm{~d} y \mathrm{~d} \mathbf{x}
$$

(recall that for the moment $t$ and $L$ are free parameters).

If we choose $L=0$, then we can see that $P_{0}$ has a kernel with well defined diagonal function $P_{0}(x, x)$ (defined via the zero energy eigenfunctions of $H$ exactly as it was done in [E-1993]), and in this case the estimate (35) can be replaced by

$$
\int_{0}^{\infty} \operatorname{Tr}\left(K_{E, L=0}^{<}\right) E^{\gamma-1} \mathrm{~d} E \leqq \frac{2^{\gamma+1}}{4 \gamma^{2}-1} \int_{\mathbf{R}^{3}}|V(\mathbf{x})|_{-}^{\gamma+1 / 2} P_{L=0}(x, x) \mathrm{d} \mathbf{x}
$$

(use directly the kernel of $P_{0}$ in (31)). Naturally, if $P_{L}(x, x)$ makes sense (or is defined as in (34)), then (36) is valid for any $L$ (simply skip the estimate (30)).

For the contribution of the upper part, for each $E>0, L \geqq 0$ we will present an operator $M_{E, L}$ which commutes with $H+p_{3}^{2}$, satisfies

$$
\left(1-\Pi_{L}\right)\left(H+p_{3}^{2}+\frac{E}{2}\right)^{-1}\left(1-\Pi_{L}\right) \leqq M_{E, L}
$$

and $M_{E, L}$ has a kernel. Then, using that $\operatorname{Tr}\left(U^{1 / 2} A U^{1 / 2}\right)^{2} \leqq \operatorname{Tr}\left(U A^{2} U\right)$ for nonnegative operators, we obtain

$$
\begin{aligned}
\operatorname{Tr}\left[\left(K_{E, L}^{>}\right)^{2}\right] & \leqq \operatorname{Tr}\left(\left|V+\frac{E}{2}\right|_{-} M_{E, L}^{2}\left|V+\frac{E}{2}\right|\right) \\
& =\int_{\mathbf{R}^{3}}\left|V(\mathbf{x})+\frac{E}{2}\right|_{-}^{2} M_{E, L}^{2}(\mathbf{x}, \mathbf{x}) \mathrm{d} \mathbf{x}
\end{aligned}
$$

As we will show later,

$$
M_{E, L}^{2}(\mathbf{x}, \mathbf{x}):=\int_{\mathbf{R}^{3}}\left|M_{E, L}(\mathbf{x}, \mathbf{y})\right|^{2} \mathrm{~d} \mathbf{y} \leqq \frac{C(L)}{\sqrt{E}}
$$

for almost all $\mathbf{x}$ and for some $L$-dependent number $C(L)$, therefore

$$
\begin{aligned}
\int_{0}^{\infty} \operatorname{Tr}\left[\left(K_{E, L}^{>}\right)^{2}\right] E^{\gamma-1} \mathrm{~d} E & \leqq C(L) \iint_{\mathbf{R}^{3}}^{\infty}\left|V(\mathbf{x})+\frac{E}{2}\right|_{-}^{2} E^{\gamma-3 / 2} \mathrm{~d} E \mathrm{~d} \mathbf{x} \\
& \leqq C(L) \frac{2^{\gamma+7 / 2}}{\left(4 \gamma^{2}-1\right)(2 \gamma+3)} \int_{\mathbf{R}^{3}}|V(\mathbf{x})|_{-}^{\gamma+3 / 2} \mathrm{~d} \mathbf{x}
\end{aligned}
$$

Combining (29), (35) and (40), we can reduce the proof of Theorems 2.1 and 2.2 to the following two propositions (recall that the diagonal elements are defined as in (34)). 
Proposition 3.1. Under the conditions of Theorem 2.1 for almost all $x$ we have

$$
e^{t L} e^{-t H}(x, x) \leqq \frac{e \cdot k_{2}^{2}(p)}{\pi}\left(L+\left\|B^{<}\right\|_{\infty}+c_{2}(p)\left\|B^{>}\right\|_{p}^{p /(p-1)}\right)
$$

with $t=\left(L+\left\|B^{<}\right\|_{\infty}+c_{2}(p)\left\|B^{>}\right\|_{p}^{p /(p-1)}\right)^{-1}$. Furthermore, for any positive $\beta$, the operator

$$
M_{E, L}:=\frac{1}{\left(1-e^{-L \beta}\right)} \int_{0}^{\beta} e^{-t\left(H+p_{3}^{2}+\frac{E}{2}\right)} \mathrm{d} t
$$

satisfies (37) and for $\beta:=\left(\left\|B^{<}\right\|_{\infty}+c_{2}(p)\left\|B^{>}\right\|_{p}^{p /(p-1)}\right)^{-1}$,

$$
M_{E, L}^{2}(\mathbf{x}, \mathbf{x}) \leqq \frac{C(L)}{\sqrt{E}} \quad \text { with } \quad C(L):=\frac{12 e^{2} \sqrt{2} k_{2}^{2}(p)}{(4 \pi)^{3 / 2}\left(1-e^{-L \beta}\right)^{2}}
$$

for almost all $\mathbf{x}$.

Remark. After proving this proposition, we choose $L:=\mu \beta^{-1}$ to finish the proof of Theorem 2.1.

Proposition 3.2. Under the conditions of Theorem 2.2 there are two constants $C_{1}$ and $C_{2}$ depending only on $c$, and there is an operator $M_{E, 0}$ satisfying (37) (with $L=0$ ) such that

$$
P_{0}(x, x) \leqq C_{1} \cdot B(x)
$$

and

$$
M_{E, 0}^{2}(\mathbf{x}, \mathbf{x}) \leqq \frac{C_{2}}{\sqrt{E}},
$$

which imply Theorem 2.2 via (29), (36) and (40).

For the proof of Theorem 2.4, we use a different idea (invented in [LSY-III]) to split the Birman-Schwinger kernel and to separate the external potential. Since the magnetic field has variable direction, we cannot decompose $\mathbf{H}_{\text {Pauli }}$ into two operators acting on $L^{2}\left(\mathbf{R}^{3}\right)$. Let $\boldsymbol{\Pi}_{L}$ be the spectral projection of $\mathbf{H}_{\text {Pauli }}^{0}:=[(\mathbf{p}-\mathbf{A}) \cdot \sigma]^{2}$ onto $[0, L]$, and recall that $\boldsymbol{\Pi}_{L}$, as well as $\mathbf{H}_{\text {Pauli }}^{0}$, acts on $L^{2}\left(\mathbf{R}^{3}, \mathbf{C}^{2}\right)$. Define the BirmanSchwinger kernel of the full Pauli operator $(E>0)$

$$
\mathbf{K}_{E}:=\left|V+\frac{E}{2}\right|_{-}^{1 / 2}\left(\mathbf{H}_{\text {Pauli }}^{0}+\frac{E}{2}\right)^{-1}\left|V+\frac{E}{2}\right|_{-}^{1 / 2},
$$

where we conveniently omitted the identity matrices, but the operator of multiplication by a function $U$ on $\mathbf{R}^{3}$ should be understood as $U \cdot \mathbf{I}$ acting on $L^{2}\left(\mathbf{R}^{3}, \mathbf{C}^{2}\right)$. As before, let $\mathbf{K}_{E}=\mathbf{K}_{E, L}^{<}+\mathbf{K}_{E, L}^{>}$, where

$$
\begin{gathered}
\mathbf{K}_{E, L}^{<}:=\left|V+\frac{E}{2}\right|_{-}^{1 / 2} \boldsymbol{\Pi}_{L}\left(\mathbf{H}_{\text {Paul }}^{0}+\frac{E}{2}\right)^{-1} \boldsymbol{\Pi}_{L}\left|V+\frac{E}{2}\right|_{-}^{1 / 2}, \\
\mathbf{K}_{E, L}^{>}:=\left|V+\frac{E}{2}\right|_{-}^{1 / 2}\left(\mathbf{I}-\boldsymbol{\Pi}_{L}\right)\left(\mathbf{H}_{\text {Pauli }}^{0}+\frac{E}{2}\right)^{-1}\left(\mathbf{I}-\boldsymbol{\Pi}_{L}\right)\left|V+\frac{E}{2}\right|_{-}^{1 / 2} .
\end{gathered}
$$


Instead of (28), we use the following idea (see [LSY-III]) to estimate $\mathbf{N}_{E}$, the number of eigenvalues of $\mathbf{H}_{\text {Pauli }}$ less than $-E$ :

$$
\begin{aligned}
\mathbf{N}_{E} & =\#\left\{\text { ev.'s of } \mathbf{K}_{\mathrm{E}} \text { bigger than } 1\right\} \\
& \leqq \#\left\{\text { ev.'s of } \mathbf{K}_{E, L}^{<} \text {bigger than } 1 / 2\right\}+\left\{\text { ev.'s of } \mathbf{K}_{E, L}^{>} \text {bigger than } 1 / 2\right\} \\
& \leqq \#\left\{\text { ev.'s of }\left|V+\frac{E}{2}\right|_{-}^{1 / 2} \boldsymbol{\Pi}_{L}\left|V+\frac{E}{2}\right|_{-}^{1 / 2} \text { bigger than } E / 4\right\}+4 \operatorname{Tr}\left(\left[\mathbf{K}_{E, L}^{>}\right]^{2}\right) \\
& \leqq \#\left\{\text { ev.'s of } \Pi_{L}|V|-\Pi_{L} \text { bigger than } E / 4\right\}+4 \operatorname{Tr}\left(\left[\mathbf{K}_{E, L}^{>}\right]^{2}\right),
\end{aligned}
$$

where we have used that the positive spectrum of $X^{*} X$ and $X X^{*}$ are the same. Therefore,

$$
\begin{aligned}
& \sum_{i}\left|E_{i, P}\right|^{\gamma}=\gamma \int_{0}^{\infty} \mathbf{N}_{E} E^{\gamma-1} \mathrm{~d} E \\
& \leqq 4^{\gamma} \gamma \operatorname{Tr}\left(\boldsymbol{\Pi}_{L}|V|-\boldsymbol{\Pi}_{L}\right)^{\gamma}+4 \gamma \int_{0}^{\infty} \operatorname{Tr}\left(\left[\mathbf{K}_{E, L}^{>}\right]^{2}\right) E^{\gamma-1} \mathrm{~d} E \\
& \leqq 4^{\gamma} \gamma \operatorname{Tr}\left(\boldsymbol{\Pi}_{L}|V|_{-}^{\gamma} \boldsymbol{\Pi}_{L}\right)+4 \gamma \int_{0}^{\infty} \operatorname{Tr}\left(\left[\mathbf{K}_{E, L}^{>}\right]^{2}\right) E^{\gamma-1} \mathrm{~d} E \\
& \leqq 4^{\gamma} \gamma \operatorname{Tr}\left(|V|_{-}^{\gamma / 2} \boldsymbol{\Pi}_{L}|V|_{-}^{\gamma / 2}\right)+4 \gamma \int_{0}^{\infty} \operatorname{Tr}\left(\left[\mathbf{K}_{E, L}^{>}\right]^{2}\right) E^{\gamma-1} \mathrm{~d} E
\end{aligned}
$$

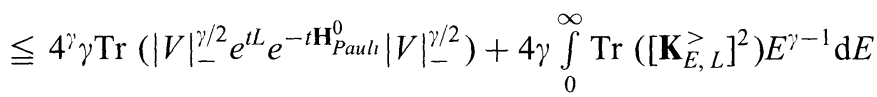

for any positive $t$, using that $\boldsymbol{\Pi}_{L} \leqq e^{t L} \exp \left(-t \mathbf{H}_{\text {Pauli }}^{0}\right)$. Note that we have used $\operatorname{Tr}(U A U)^{\gamma} \leqq \operatorname{Tr}\left(U^{\gamma} A^{\gamma} U^{\gamma}\right)$ which requires $\gamma \geqq 1$ (while Theorems 2.1 and 2.2 required only $\gamma>1 / 2$ ).

We will again present an operator $\mathbf{M}_{E, L}$, commuting with $\mathbf{H}_{\text {Paull }}^{0}$, such that

$$
\left(\mathbf{I}-\boldsymbol{\Pi}_{L}\right)\left(\mathbf{H}_{\text {Paull }}^{0}+\frac{E}{2}\right)^{-1}\left(\mathbf{I}-\boldsymbol{\Pi}_{L}\right) \leqq \mathbf{M}_{E, L},
$$

then following (38) and (40), the proof of Theorem 2.4 is reduced to the following proposition.

Proposition 3.3. Under the conditions of Theorem 2.4,

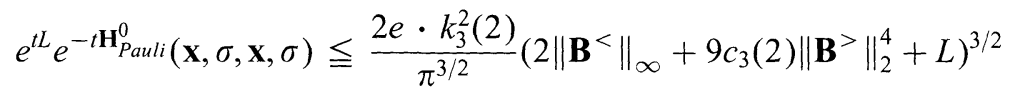

$\left(\mathbf{x} \in \mathbf{R}^{3}\right.$ and $\left.\sigma \in\{0,1\}\right)$ for $t:=\left(2\left\|\mathbf{B}^{<}\right\|_{\infty}+9 c_{3}(2)\left\|\mathbf{B}^{>}\right\|_{2}^{4}+L\right)^{-1}$. Furthermore,

$$
\mathbf{M}_{E, L}:=\frac{1}{1-e^{-L \beta}} \int_{0}^{\beta} e^{-t\left(\mathbf{H}_{\text {Paul }}^{0}+\frac{E}{2}\right)} \mathrm{d} t
$$


satisfies (51) and for $\beta:=\left(2\left\|\mathbf{B}^{<}\right\|_{\infty}+9 c_{3}(2)\left\|\mathbf{B}^{>}\right\|_{2}^{4}\right)^{-1}$,

$$
\mathbf{M}_{E, L}^{2}(\mathbf{x}, \sigma, \mathbf{x}, \sigma) \leqq \frac{C^{\prime}(L)}{\sqrt{E}} \quad \text { with } C^{\prime}(L):=\frac{12 \sqrt{2} e^{2} \cdot k_{3}^{2}(2)}{\pi^{3 / 2}\left(1-e^{-L \beta}\right)^{2}} .
$$

In case of $\mathbf{B}^{>}=0$, the factor $k_{3}(2)$ can be replaced by 1 everywhere.

\section{Feynman-Kac Formulas}

4.1. Two-Dimensional Case. The proofs of Propositions 3.1 and 3.2 rely on the magnetic Feynman-Kac formula for the two-dimensional Pauli operator. Let $\mathbf{E}_{0, x}^{2 t, y}$ denote the expectation for the two-dimensional Brownian bridge $W(s)(0 \leqq s \leqq 2 t)$ under the conditions $W(0)=x$ and $W(2 t)=y$.

Proposition 4.1. Let $A$ be a vectorfield on $\mathbf{R}^{2}$ such that $A \in L_{l o c}^{2}$, $\operatorname{div} A \in L_{l o c}^{2}$ and $B:=\operatorname{rot} A \in L_{\text {loc }}^{2}$. Furthermore, we assume that the following conditions are satisfied:

(i) either $B=B^{<}+B^{>}$with $B^{<} \in L^{p}$ (for $\left.p>1\right)$ and $B^{>} \geqq 0\left(B^{>} \geqq 0\right.$ can be dropped for $p=2$ );

(ii) or $A$ and $\operatorname{div} A$ do not grow faster than some polynomial at infinity, furthermore let $B:=\operatorname{rot} A \in C^{1}$ and assume that $0 \leqq B(x) \leqq c_{0}\left(|x|^{2-\varepsilon}+1\right)$ with some positive $\varepsilon$ and $c_{0}$.

Then the heat operator $\exp (-t H)$ of the two-dimensional operator $H:=(p-$ $A)^{2}-B$ has a kernel $D^{(t)}(x, y)$ defined by

$$
D^{(t)}(x, y):=\frac{1}{4 \pi t} \cdot e^{-\frac{(x-y)^{2}}{4 t}} \mathbf{E}_{0, x}^{2 t, y} \exp \Psi_{A, B}(W),
$$

where

$$
\begin{aligned}
\Psi_{A, B}(W) & :=-i \int_{0}^{2 t} A(W(s)) \circ \mathrm{d} W(s)+\frac{1}{2} \int_{0}^{2 t} B(W(s)) \mathrm{d} s \\
& =-i \int_{0}^{2 t} A(W(s)) \mathrm{d} W(s)+\frac{i}{2} \int_{0}^{2 t} \operatorname{div} A(W(s)) \mathrm{d} s+\frac{1}{2} \int_{0}^{2 t} B(W(s)) \mathrm{d} s
\end{aligned}
$$

(as usual, $\int F(W) \mathrm{d} W$ denotes the Ito integral, while $\int F(W) \circ \mathrm{d} W$ is the Stratonovich integral). Under conditions (i) for almost all $x$

$$
e^{-t H}(x, x):=\int_{\mathbf{R}^{2}}\left|D^{(t / 2)}(x, y)\right|^{2} \mathrm{~d} y \leqq \frac{k_{2}^{2}(p) \cdot \exp \left[t\left(\left\|B^{<}\right\|_{\infty}+c_{2}(p)\left\|B^{>}\right\|_{p}^{p /(p-1)}\right)\right]}{\pi t}
$$

$\left(k_{2}(p)\right.$ can be replaced by 1 if $\left.B^{>}=0\right)$. Under conditions (ii) there exists $a$ continuous function $\Gamma(t, x):[0, \infty) \times \mathbf{R}^{2} \rightarrow \mathbf{R}_{+}$such that

$$
\left|D^{(t)}(x, y)\right| \leqq \frac{\Gamma(t, x)}{t} \cdot e^{-\frac{(x-y)^{2}}{8 t}}
$$


If, in addition, $B$ is continuously differentiable, and $A$ and $\operatorname{div} A$ are continuous then $D^{(t)}(x, y)$ is continuous and $e^{-t H}$ maps $L^{2}\left(\mathbf{R}^{2}\right)$ into $C\left(\mathbf{R}^{2}\right)$.

Remark. 1. The kernel of the heat operator is defined only almost everywhere, while $D^{(t)}(x, y)$ is defined for all $x, y$. What we are going to prove is that $D^{(t)}(x, y)$, as defined above, can be a realization of the heat kernel.

Remark. 2. If we know that $A$ and $\operatorname{div} A$ are continuous, then it is enough to assume that $B$ is continuous for the continuity result. But usually only $B$ is given, and then for choosing a continuous gauge with continuous divergence we had better assume $B \in C^{1}$.

Remark. 3. Under the conditions (i) (actually much less is needed), the continuity of the heat kernel $\exp (-t H)(x, y)$ in $t, x, y$ is proved in [BHL]. We do not want to use this result here (and therefore we keep on defining the diagonal element as in (34)), since we shall apply a parallel argument for the full Pauli operator $\mathbf{H}_{\text {Pauli }}^{0}$, where the continuity of the heat kernel has not yet been established. The continuity result in Proposition 4.1 under the conditions (ii) is not covered by [BHL].

Proof. The proof of this proposition under the conditions (ii) is found in [E-1993]. The only difference between this proposition and the statement in Appendix B of [E$1993]$ is that in (58) we take into account the $1 / t$ singularity, so $\Gamma(t, x)$ is continuous for $t \geqq 0$, while the corresponding function in Eq. (71) in [E-1993] was continuous only for $t>0$.

Now let us consider the conditions (i). First we show that the expectation value in (55) is absolutely convergent. Let $\mathscr{H}:=-\Delta-B$ acting on $L^{2}\left(\mathbf{R}^{2}\right)$, then by the usual Feynman-Kac formula $\exp (-t \mathscr{H})$ has a continuous kernel (see [S-1982]), defined as

$$
e^{-t \mathscr{H}}(x, y):=\frac{1}{4 \pi t} \cdot e^{\frac{(x-y)^{2}}{4 t}} \mathbf{E}_{0, x}^{2 t, y} e^{\frac{1}{2} \int_{0}^{2 t} B(W(s)) \mathrm{d} s},
$$

which, in particular, shows that the expectation on the right-hand side of (59) is finite for all $x, y$. Then obviously the right-hand side of (55) is well defined and finite.

Next we prove the estimate (57) (notice that the diagonal elements exp $(-t H)$ $(x, x)$ are defined separately as in (34)). For the heat kernel of $\mathscr{H}$ we have

$$
\begin{aligned}
e^{-t \mathscr{H}}(x, x)= & \int_{\mathbf{R}^{2}} e^{-t \mathscr{H} / 2}(x, y) e^{-t \mathscr{H} / 2}(y, x) \mathrm{d} y \\
& =\left(e^{-t \mathscr{H} / 2} f_{x}\right)(x) \\
\leqq & \left\|e^{-t \mathscr{H} / 2}\right\|_{1, \infty}\left\|f_{x}\right\|_{1} \leqq\left\|e^{-t \mathscr{H} / 2}\right\|_{1, \infty}\left\|e^{-t \mathscr{H} / 2}(1)\right\|_{\infty} \\
& \leqq\left\|e^{-t \mathscr{H} / 2}\right\|_{1, \infty}\left\|e^{-t \mathscr{H} / 2}\right\|_{\infty, \infty}
\end{aligned}
$$

with $f_{x}(y):=e^{-t \mathscr{H} / 2}(y, x)$ (here $\|\cdot\|_{p, q}$ denotes the operator norm from $L^{p}$ to $L^{q}$ ). Using Carmona's bounds (see Proposition 3.1 and Remark 3.1 in [C]) one obtains

$$
e^{-t \mathscr{H}}(x, x) \leqq \frac{k_{2}^{2}(p)}{\pi t} \cdot \exp \left[t\left(\left\|B^{<}\right\|_{\infty}+c_{2}(p)\left\|B^{>}\right\|_{p}^{p /(p-1)}\right)\right] .
$$


Using that $\left|D^{(t / 2)}(x, y)\right| \leqq e^{-t \mathscr{H} / 2}(x, y)$ for almost all $x, y,(57)$ follows.

To prove that $D^{(t)}(x, y)$ is actually the heat kernel, first recall that for $A \in L_{l o c}^{2}$, $\operatorname{div} A \in L_{l o c}^{2}$ and a bounded function $U$ we have (in an almost everywhere sense)

$$
e^{-t\left[(p-A)^{2}-U\right]}(x, y)=\frac{1}{4 \pi t} \cdot e^{-\frac{(x-y)^{2}}{4 t}} \mathbf{E}_{0, x}^{2 t, y} \Psi_{A, U}(W)
$$

(see $[\mathrm{S}-1979(\mathrm{a})])$. For $B^{>} \geqq 0$ let $U_{n}:=B^{<}+\min \left(n, B^{>}\right)$be an increasing sequence of functions, then (62) is valid for $U_{n}$. When taking $n \rightarrow \infty$ limit, the righthand side converges to the right-hand side of (55), using the dominated convergence theorem on the path space and the finiteness of (59). As to the left-hand side, it is enough to show that $\exp \left(-t H_{n}\right) \rightarrow \exp (-t H)$ strongly, where $H_{n}:=(p-A)^{2}-U_{n}$ (here $H_{n}$ is not a Pauli operator, since $U_{n} \neq \operatorname{rot} A$, but $U$ is independent of $A$ in (62)). Using that $H_{n} \searrow H \geqq 0$, we conclude that $H_{n} \rightarrow H$ in a strong resolvent sense (see [K, VIII. 3.11]), which, in particular, implies the strong convergence of the heat kernels.

If $p=2$ and $B^{>}$has no definite sign, then instead of the last paragraph one uses the same argument as in the proof of Proposition 4.2 below to show that $D^{(t)}(x, y)$ is really the heat kernel.

4.2.Three-Dimensional Case. For the proof of Proposition 3.3, we need a FeynmanKac formula for the three-dimensional Pauli operator $\mathbf{H}_{\text {Pauli }}^{0}$. The expectation of the three-dimensional Brownian bridge $\mathbf{W}(s)$, with constraints $\mathbf{W}(0)=\mathbf{x}, \mathbf{W}(2 t)=\mathbf{y}$, is denoted by $\mathbf{E}_{0, \mathbf{x}}^{2 t, \mathbf{y}}$. We shall also need a pure Poisson process $v(s)$ with unit jump rate $(\mathbf{E d} v(s)=\mathrm{d} s)$ and with initial value $v(0)=0$ or $v(0)=1$. Let $\bar{v}(s)$ denote $v(s)$ modulo 2, which is a Poisson process on $\mathbf{Z}_{2}$. The "Poisson bridge" is defined as a Poisson process $v(s)$ with endpoints satisfying $v(0)=\sigma$ and $\bar{v}(2 t)=\sigma^{\prime}$ for some fixed $\sigma, \sigma^{\prime} \in\{0,1\}$. The corresponding expectation is denoted by $\mathbf{E}_{0, \sigma}^{2 t, \sigma^{\prime}}$ (it depends only $\left.\left|\sigma-\sigma^{\prime}\right|\right)$.

Proposition 4.2. Let $\mathbf{A}$ be a vectorfield on $\mathbf{R}^{3}$, such that $\mathbf{A} \in L_{l o c}^{2}\left(\mathbf{R}^{3}\right)$ and $\operatorname{div} \mathbf{A} \in$ $L_{\text {loc }}^{2}\left(\mathbf{R}^{3}\right)$. Furthermore, let the vectorfield $\mathbf{B}=\left(B_{1}, B_{2}, B_{3}\right)$ admit a decomposition $\mathbf{B}=\mathbf{B}^{<}+\mathbf{B}^{>}$such that $\mathbf{B}^{<} \in L^{\infty}\left(\mathbf{R}^{3}\right)$ and $\mathbf{B}^{>} \in L^{2}\left(\mathbf{R}^{3}\right)$. (For $\mathbf{B}:=\operatorname{rot} \mathbf{A}$ we will get back the Pauli operator, but this relation is not necessary for the present statement. ) Then

$$
\exp \left(-t\left[(\mathbf{p}-\mathbf{A})^{2}-\boldsymbol{\sigma} \cdot \mathbf{B}\right]\right)\left(\text { in particular } \exp \left(-t \mathbf{H}_{\text {Pauli }}^{0}\right)\right)
$$

has a kernel $\mathbf{D}^{(t)}\left(\mathbf{x}, \sigma, \mathbf{y}, \sigma^{\prime}\right)$ defined as

$$
\mathbf{D}^{(t)}\left(\mathbf{x}, \sigma, \mathbf{y}, \sigma^{\prime}\right):=\frac{e^{-2 t}\left(\cosh (2 t)-e^{-2 t}\left(\sigma-\sigma^{\prime}\right)^{2}\right)}{(4 \pi t)^{3 / 2}} \cdot e^{-\frac{(\mathbf{x}-\mathbf{y})^{2}}{4 t}} \mathbf{E}_{0, \sigma}^{2 t, \sigma^{\prime}} \mathbf{E}_{0, \mathbf{x}}^{2 t, \mathbf{y}} \boldsymbol{\Psi}_{\mathbf{A}, \mathbf{B}}(\mathbf{W}, v),
$$

where

$$
\begin{aligned}
\boldsymbol{\Psi}_{\mathbf{A}, \mathbf{B}}(\mathbf{W}, v):=e^{2 t} \exp ( & -i \int_{0}^{2 t} \mathbf{A}(\mathbf{W}(s)) \circ \mathrm{d} \mathbf{W}(s)+\frac{1}{2} \int_{0}^{2 t} B_{3}(\mathbf{W}(s))(-1)^{v(s)} \mathrm{d} s \\
& \left.+\int_{0}^{2 t} \log \left[\frac{1}{2}\left(B_{1}(\mathbf{W}(s))-i(-1)^{v(s)} B_{2}(\mathbf{W}(s))\right)\right] \mathrm{d} v(s)\right) .
\end{aligned}
$$


Furthermore, we have for the diagonal elements

$$
\begin{aligned}
\left|\mathbf{D}^{(t)}(\mathbf{x}, \sigma, \mathbf{x}, \sigma)\right| & :=\left|\sum_{\sigma^{\prime}} \int_{\mathbf{R}^{3}} \mathbf{D}^{(t / 2)}\left(\mathbf{x}, \sigma, \mathbf{y}, \sigma^{\prime}\right) \mathbf{D}^{(t / 2)}\left(\mathbf{y}, \sigma^{\prime}, \mathbf{x}, \sigma\right) \mathrm{d} \mathbf{y}\right| \\
& \leqq \frac{2 k_{3}^{2}(2) \cdot \exp \left[t\left(2\left\|\mathbf{B}^{<}\right\|_{\infty}+9 c_{3}(2)\left\|\mathbf{B}^{>}\right\|_{2}^{4}\right)\right]}{(\pi t)^{3 / 2}}
\end{aligned}
$$

(and $k_{3}(2)$ can be replaced by 1 if $\mathbf{B}^{>}=0$ ).

Remark. The unusual factor in (64)

$$
\begin{aligned}
Q\left(\sigma, \sigma^{\prime}, 2 t\right): & =e^{-2 t}\left(\cosh (2 t)-e^{-2 t}\left(\sigma-\sigma^{\prime}\right)^{2}\right) \\
& =e^{-2 t}\left(\cosh (2 t) \cdot \mathbf{1}\left(\sigma=\sigma^{\prime}\right)+\sinh (2 t) \cdot \mathbf{1}\left(\sigma \neq \sigma^{\prime}\right)\right) \\
& =\operatorname{Prob}\left\{(-1)^{\sigma^{\prime}}=(-1)^{v(2 t)+\sigma}\right\}=\operatorname{Prob}\left\{\bar{v}(2 t)=\sigma^{\prime} \mid v(0)=\sigma\right\}
\end{aligned}
$$

comes from the normalization of the Poisson bridge: $\mathbf{E}_{0, \sigma}^{2 t, \sigma^{\prime}}(1)=1$.

Proof. The proof is basically given in [DJS], where it is proved that

$$
f_{t}(\mathbf{x}, \sigma):=\sum_{\sigma^{\prime}} \int_{\mathbf{R}^{3}} \mathbf{D}^{(t)}\left(\mathbf{x}, \sigma, \mathbf{y}, \sigma^{\prime}\right) f_{0}\left(\mathbf{y}, \sigma^{\prime}\right) \mathrm{d} \mathbf{y}
$$

satisfies $\mathrm{d} f_{t} / \mathrm{d} t=-\left[(\mathbf{p}-\mathbf{A})^{2}-\boldsymbol{\sigma} \cdot \mathbf{B}\right] f_{t}$ with the initial condition $f_{0}$, i.e.

$$
f_{t}=e^{-t\left[(\mathbf{p}-\mathbf{A})^{2}-\sigma \cdot \mathbf{B}\right]} f_{0} .
$$

The calculation given in Appendix 2 of [DJS] is rigorous for smooth $\mathbf{A}$ and $\mathbf{B}$. (Except that the divergence term is omitted, but it can obviously be incorporated into the potential term. Probably the authors assumed that the vector potential is divergence free, although they did not say so.) For the Ito calculus extended to the joint Wiener-Poisson process, it is enough if all data and $f_{t}(\mathbf{x}, \sigma)$ are $C^{2}$ (see [GS]). To prove the smoothness of $f_{t}$, notice that for any fixed realization of the Poisson process the function

$$
(\mathbf{x}, \mathbf{y}) \rightarrow \mathbf{E}_{0, \mathbf{x}}^{2 t, \mathbf{y}} \boldsymbol{\Psi}(\mathbf{W}, v)
$$

is smooth using an argument similar to Appendix B of [E-1993] and the Taylor expansion of the data. Moreover, the estimates are uniform in $v$, so we conclude that $\mathbf{D}^{(t)}$, the average of the functions $(70)$ is also smooth.

To justify that (64) is really the heat kernel for more general data, first we show that the double expectation on the right-hand side (64) is absolutely convergent. Clearly

$$
\begin{aligned}
& Q\left(\sigma, \sigma^{\prime}, 2 t\right) \mathbf{E}_{0, \sigma}^{2 t, \sigma^{\prime}} \mathbf{E}_{0, \mathbf{x}}^{2 t, \mathbf{y}}\left|\Psi_{\mathbf{A}, \mathbf{B}}(\mathbf{W}, v)\right| \\
& \leqq Q\left(\sigma, \sigma^{\prime}, 2 t\right) e^{2 t} \mathbf{E}_{0, \mathbf{x}}^{2 t, \mathbf{y}} \mathbf{E}_{0, \sigma}^{2 t, \sigma^{\prime}} \\
& \times \exp \left\{\frac{1}{2} \int_{0}^{2 t}\left|B_{3}(\mathbf{W}(s))\right| \mathrm{d} s+\int_{0}^{2 t} \log \frac{1}{2}\left(B_{1}^{2}(\mathbf{W}(s))+B_{2}^{2}(\mathbf{W}(s))\right)^{1 / 2} \mathrm{~d} v(s)\right\} \\
& \leqq \mathbf{E}_{0, \mathbf{x}}^{2 t, \mathbf{y}} e^{\int_{0}^{2 t}|\mathbf{B}(\mathbf{W}(s))| \mathrm{d} s},
\end{aligned}
$$


using that for the exponential moment of the Poisson integral we have (with the convention that $e^{-\infty}=0$ )

$$
\mathbf{E}_{0, \sigma}^{2 t, \sigma} \exp \left(\int_{0}^{2 t} \log F(\tau) \mathrm{d} v(\tau)\right)=\frac{\cosh \left(\int_{0}^{2 t} F(\tau) \mathrm{d} \tau\right)}{\cosh 2 t}
$$

and a similar expression for $\mathbf{E}_{0, \sigma}^{2 t, \sigma^{\prime}} \ldots$ if $\sigma \neq \sigma^{\prime}$, but then one has sinh's instead of cosh's on the right-hand side of (72). For a step-function $F$, these formulae easily follow from the basic addition rules of the cosh and sinh, and from the fact that

$$
\mathbf{E}_{0, \sigma}^{2 t, \sigma} e^{\chi(v(2 t)-v(0))}=Q(\sigma, \sigma, 2 t)^{-1} \sum_{n=0}^{\infty} e^{2 n \alpha} \cdot \frac{e^{-2 t}(2 t)^{2 n}}{(2 n) !}=\frac{\cosh \left(2 t e^{\alpha}\right)}{\cosh 2 t}
$$

and similarly

$$
\mathbf{E}_{0, \sigma}^{2 t, \sigma^{\prime}} e^{\alpha(v(2 t)-v(0))}=\frac{\sinh \left(2 t e^{\alpha}\right)}{\sinh 2 t}
$$

for $\sigma \neq \sigma^{\prime}$. For a general measurable nonnegative function $F$, use a limiting argument.

Let $\mathscr{H}:=-\Delta-2|\mathbf{B}|$ on $L^{2}\left(\mathbf{R}^{3}\right)$, then the heat kernel is

$$
e^{-t \mathscr{H}}(\mathbf{x}, \mathbf{y})=\frac{1}{(4 \pi t)^{3 / 2}} \cdot e^{-\frac{(\mathbf{x}-\mathbf{y})^{2}}{4 t}} \mathbf{E}_{0, \mathbf{x}}^{2 t, \mathbf{y}} e^{\int_{0}^{2 t}|\mathbf{B}(\mathbf{W}(s))| \mathrm{d} s}
$$

by the usual Feynman-Kac formula and it is continuous (see [S-1982]). In particular, (75) is finite for all $\mathbf{x}, \mathbf{y}$, which, together with (71), shows that $\mathbf{D}^{(t)}\left(\mathbf{x}, \sigma, \mathbf{y}, \sigma^{\prime}\right)$ is well-defined for almost all $\mathbf{x}, \mathbf{y}$. To prove (66) we use (see (71))

$$
\left|\mathbf{D}^{(t / 2)}\left(\mathbf{x}, \sigma, \mathbf{y}, \sigma^{\prime}\right)\right| \leqq e^{-t \mathscr{H} / 2}(\mathbf{x}, \mathbf{y})
$$

(for almost all $\mathbf{x}, \mathbf{y}$ ) and Carmona's bounds. The calculation is similar to (60) and (61).

Now let us given general data $\left(\mathbf{A}, \operatorname{div} \mathbf{A} \in L_{l o c}^{2}, \mathbf{B}=\mathbf{B}^{<}+\mathbf{B}^{>}\right.$, with $\mathbf{B}^{<} \in L^{\infty}$, $\mathbf{B}^{>} \in L^{2}$ ), then one can choose a sequence of smooth data $\mathbf{A}_{n}$ and $\mathbf{B}_{m}=\mathbf{B}_{m}^{<+} \mathbf{B}_{m}^{>}$, such that $\mathbf{A}_{n} \rightarrow \mathbf{A}$ in $L^{2}$ and almost everywhere (by passing to a subsequence), and the same is true for $\operatorname{div} \mathbf{A}_{n}, \mathbf{B}_{m}^{<}$and for $\mathbf{B}_{m}^{>}$with uniformly bounded $\mathbf{B}_{m}^{<}$, and bounded $\mathbf{B}_{m}^{>}$(the limits are taken independently $n, m \rightarrow \infty$ ). Note that $\mathbf{A}$, divA and $\mathbf{B}^{<}$are not necessarily in $L^{2}$, just in $L_{l o c}^{2}$, nevertheless one can approximate them by smooth functions in $L^{2}$-sense (i.e. the difference in $L^{2}$ goes to zero). Then by the dominated covergence (use (66)), the right-hand side of (64) converges (as $n, m \rightarrow$ $\infty$ independently). For the strong convergence of $\exp \left(-t\left[\left(\mathbf{p}-\mathbf{A}_{n}\right)^{2}-\boldsymbol{\sigma} \cdot \mathbf{B}_{m}\right]\right)$ (in some convenient order of the limits) it is enough to show the strong resolvent convergence (these operators are uniformly semibounded as it can be seen from the argument below). First use that for any fixed $m,\left(\mathbf{p}-\mathbf{A}_{n}\right)^{2}-\boldsymbol{\sigma} \cdot \mathbf{B}_{m}$ converges to $(\mathbf{p}-\mathbf{A})^{2}-\boldsymbol{\sigma} \cdot \mathbf{B}_{m}$ in a strong resolvent sense. This follows from Theorem 15.4 in [S-1979] about the strong convergence of $R_{n}(E):=\left(\left(\mathbf{p}-\mathbf{A}_{n}\right)^{2}+E\right)^{-1}$, and from the representation (for some large $E>\left\|\mathbf{B}_{m}\right\|_{\infty}$ )

$$
\left(\left(\mathbf{p}-\mathbf{A}_{n}\right)^{2}-\boldsymbol{\sigma} \cdot \mathbf{B}_{m}+E\right)^{-1}=\left(1-R_{n}(E) \boldsymbol{\sigma} \cdot \mathbf{B}_{m}\right)^{-1} R_{n}(E) .
$$

Finally, we have to show that $\mathbf{H}-\boldsymbol{\sigma} \cdot \mathbf{B}_{m}$ converges to $\mathbf{H}-\boldsymbol{\sigma} \cdot \mathbf{B}$ in a strong resolvent sense, where $\mathbf{H}:=(\mathbf{p}-\mathbf{A})^{2}$. Using that

$$
\left(\mathbf{H}-\boldsymbol{\sigma} \cdot \mathbf{B}_{m}+E\right)^{-1}=R(E)\left(1-\left(\boldsymbol{\sigma} \cdot \mathbf{B}_{m}\right) R(E)\right)^{-1}
$$


(where $R(E)$ is the resolvent of $\mathbf{H}$ ), it is enough to show that for some large $E$ the norm of $\left(\boldsymbol{\sigma} \cdot \mathbf{B}_{m}\right) R(E)$ is uniformly bounded by $1 / 2$, and that $\left(\boldsymbol{\sigma} \cdot \mathbf{B}_{m}\right) R(E)$ converges to $(\boldsymbol{\sigma} \cdot \mathbf{B}) R(E)$ strongly. Both statements easily follow from the facts that $\left\|\mathbf{B}_{m}-\mathbf{B}\right\|_{2} \rightarrow 0$ and that $R(E)$ maps $L^{2}$ into any $L^{q}$ with $q \geqq 2$ with arbitrarily small norm (if $E$ can be chosen large). For the contracting properties of $R(E)$ one uses the diamagnetic inequality and the corresponding statements for the resolvent of the free Laplacian.

\section{Bounded Magnetic Field with Weak Singularities}

In this section we prove Propositions 3.1 and 3.3. The first part of Proposition 3.1 (estimate (41)) immediately follows from (57).

For the other statement, first use the simple fact that for any $\beta>0$,

$$
\frac{1}{u+\frac{E}{2}} \leqq \frac{1}{1-e^{-L \beta}} \int_{0}^{\beta} e^{-t\left(u+\frac{E}{2}\right)} \mathrm{d} t
$$

If $u \geqq L$. Fix $\beta:=\left(\left\|B^{<}\right\|_{\infty}+c_{2}(p)\left\|B^{>}\right\|_{p}^{p /(p-1)}\right)^{-1}$ and apply this inequality for the spectral resolution of $H+p_{3}^{2}$, which is bigger than $L$ on the subspace $\operatorname{Ran}\left(I-\Pi_{L}\right)$. This implies immediately that $M_{E, L}$ defined in (42) satisfies (37) (since all the operators commute with $\Pi_{L}$, it is enough to check (37) on $\operatorname{Ran}\left(\Pi_{L}\right)$ and on $\operatorname{Ran}\left(I-\Pi_{L}\right)$ separately; the first is trivial, the second follows from (79)).

By Proposition 4.1, for the kernel $M_{E, L}(\mathbf{x}, \mathbf{y})$ we have

$$
\left|M_{E, L}(\mathbf{x}, \mathbf{y})\right| \leqq \frac{1}{1-e^{-L \beta}} \int_{0}^{\beta} \frac{1}{(4 \pi t)^{1 / 2}} \cdot e^{-\frac{\left(x_{3}-y_{3}\right)^{2}}{4 t}} e^{-t \frac{E}{2}}\left|D^{(t)}(x, y)\right| \mathrm{d} t .
$$

Therefore, using $\left|D^{(t)}(x, y)\right| \leqq e^{-t \mathscr{H}}(x, y)$ and (61) we have

$$
\begin{aligned}
M_{E, L}^{2}(\mathbf{x}, \mathbf{x})= & \int_{\mathbf{R}^{3}} M_{E, L}(\mathbf{x}, \mathbf{y}) M_{E, L}(\mathbf{y}, \mathbf{x}) \mathrm{d} y \\
\leqq & \frac{1}{\left(1-e^{-L \beta}\right)^{2}} \int_{0}^{\beta} \int_{0}^{\beta} \int_{\mathbf{R}^{3}} e^{-(t+s) \frac{E}{2}} e^{-t \mathscr{H}}(x, y) e^{-s \cdot H}(y, x) \\
& \times \frac{\exp \left(-\left(x_{3}-y_{3}\right)^{2}\left(\frac{1}{4 t}+\frac{1}{4 s}\right)\right)}{(4 \pi t)^{1 / 2}(4 \pi s)^{1 / 2}} \mathrm{~d} \mathbf{y} \mathrm{d} s \mathrm{~d} t \\
\leqq & \frac{1}{\left(1-e^{-L \beta}\right)^{2}} \int_{0}^{\beta} \int_{0}^{\beta} e^{-(t+s) \frac{E}{2}} \cdot \frac{1}{(4 \pi(t+s))^{1 / 2}} \\
& \times e^{-(t+s) \mathscr{H}}(x, x) \mathrm{d} s \mathrm{~d} t \\
\leqq & \frac{4 e^{2} k_{2}^{2}(p)}{\left(1-e^{-L \beta}\right)^{2}} \int_{0}^{\beta} \int_{0}^{\beta} e^{-(t+s) \frac{E}{2}} \frac{1}{(4 \pi(t+s))^{3 / 2}} \mathrm{~d} s \mathrm{~d} t \\
\leqq & \frac{4 \sqrt{2} e^{2} k_{2}^{2}(p)}{(4 \pi)^{3 / 2}\left(1-e^{-L \beta}\right)^{2}} \cdot \frac{1}{\sqrt{E}} \int_{0}^{\infty} \frac{e^{-v}}{\sqrt{v}} \mathrm{~d} v .
\end{aligned}
$$

The last integral is estimated by 3 to obtain (43). 
The proof of Proposition 3.3 is very similar to the previous proof, we just have to use Proposition 4.2 instead of Proposition 4.1. Inequality (52) is obvious from (66). Finally, the proof of (54) is completely analogous to the second part of the proof of Proposition 3.1 using

$$
\left|\mathbf{M}_{E, L}\left(\mathbf{x}, \sigma, \mathbf{y}, \sigma^{\prime}\right)\right| \leqq \frac{1}{1-e^{-\beta L}} \int_{0}^{\beta} e^{-t \frac{E}{2}} e^{-t \mathscr{H}}(\mathbf{x}, \mathbf{y}) \mathrm{d} t
$$

with $\beta:=\left(2\left\|\mathbf{B}^{<}\right\|_{\infty}+9 c_{3}(2)\left\|\mathbf{B}^{>}\right\|_{2}^{4}\right)^{-1}$.

\section{Unbounded Magnetic Field; Reduction to the Main Lemma}

In the rest of the paper we present the proof of Proposition 3.2. In Sect. 5 we have heavily used the essentially global boundedness of $B$ (with some weak singularities), since this allowed us to estimate the magnetic heat kernel in the most trivial way (diamagnetic inequality and estimating the $\int B(W(s)) \mathrm{d} s$ term basically by the supremum norm, or by Carmona's bounds in case of the singularities). If we do not want to assume essential boundedness, or we wish to obtain the "real" estimate (4) instead of (7), then we have to analyze the local behavior of $H=(p-A)^{2}-B$.

Lower part of the spectrum; proof of (44). The first inequality in Proposition 3.2 is a straightforward consequence of Lemma 6.1 below. At this point we still do not make use of the oscillation effect in the magnetic Feynman-Kac formula due to the $-i \int A \circ \mathrm{d} W$ term. The proof of the second inequality (45) is much more difficult because we have to exploit the full power of this oscillation. We will compare the heat kernel of $H$ with that of the operator with constant magnetic field. This is the content of the Main Lemma 6.2, formulated at the end of this section.

First, we prove the following technical estimate which will be used throughout our stochastic analysis.

Lemma 6.1. Let $F: \mathbf{R}^{2} \rightarrow \mathbf{R}$ be a measurable function with $|F(w)| \leqq d|w|$, furthermore assume that $0<t \leqq 1 / B_{0}, d \leqq c B_{0}^{3 / 2}$ for some positive $B_{0}$ and $c$. Then there exist two constants $C^{(0)}=C^{(0)}(c)$ and $C^{(1)}=C^{(1)}(c)$ depending only on $c$ such that the following estimates hold for $z \in \mathbf{R}^{2}$ :

$$
\mathbf{E}_{0,0}^{2 t, z} \exp \left(\frac{1}{2} \int_{0}^{2 t} F(W(s)) \mathrm{d} s\right) \leqq C^{(0)} \exp \left(\frac{z^{2}}{40 t}\right)
$$

and

$$
\mathbf{E}_{0,0}^{2 t, z}\left(\frac{1}{2 t} \int_{0}^{2 t} F(W(s)) \mathrm{d} s\right) \exp \left(\frac{1}{2} \int_{0}^{2 t} F(W(s)) \mathrm{d} s\right) \leqq C^{(1)} d\left(1+t^{2}\right) \exp \left(\frac{z^{2}}{20 t}\right) .
$$

Proof. Consider the absolute value process $r(s):=|W(s)|$ (Bessel process), and use the upper bound for $|F|$ to transform (83) and (84) into inequalities on $r(s)$. There is an explicit formula for the exponential moment of the integral of $r^{2}(s)$, so we 
estimate $r(s)$ from above by $K+M r^{2}(s)$, where $K:=100 t^{2} d / \pi^{2}$ and $M:=1 /(4 K)$. Therefore

$$
\begin{aligned}
\mathbf{E}_{0,0}^{2 t, z} \exp \left(\frac{1}{2} \int_{0}^{2 t} F(W(s)) \mathrm{d} s\right) & \leqq e^{K d t} \cdot \mathbf{E} \exp \left(\frac{b^{2}}{2} \int_{0}^{2 t} r^{2}(s) \mathrm{d} s\right) \\
& =e^{K d t} \cdot \frac{2 b t}{\sin 2 b t} \exp \left(\frac{z^{2}}{4 t}(1-2 b t \cot (2 b t))\right),
\end{aligned}
$$

where $b:=\sqrt{M d} \leqq \frac{\pi}{20 t}$, i.e. $2 b t \leqq \pi / 10$, and $\mathbf{E}$ denotes the expectation for the process $r(s)$. Here we used the analytic extension of the Laplace transform of $\int_{0}^{2 t} r^{2}(s) d s$ given, for example, in [Y, p.17] or in [E-1994(a)]. The analytic extension is possible for $2 b t<\pi$. Using that $(1-2 b t \cot (2 b t)) \leqq 1 / 10$ and $2 b t \leqq 2 \sin (2 b t)$ for $2 b t \leqq \pi / 10$, we easily obtain (83).

For (84), one applies Hölder's inequality,

$$
\begin{aligned}
\mathbf{E}_{0,0}^{2 t, z} & \left(\frac{1}{2 t} \int_{0}^{2 t} F(W(s)) \mathrm{d} s\right) \exp \left(\frac{1}{2} \int_{0}^{2 t} F(W(s)) \mathrm{d} s\right) \\
\leqq & \frac{d}{2} \mathbf{E}_{0,0}^{2 t, z}\left(1+\left(\frac{1}{2 t} \int_{0}^{2 t}|W(s)| \mathrm{d} s\right)^{2}\right) e^{\frac{d}{2} \int_{0}^{2 t}|W(s)| \mathrm{d} s} \\
\leqq & \frac{C^{(0)} d}{2} \exp \left(\frac{z^{2}}{40 t}\right)+\frac{d}{2} \mathbf{E}\left(\frac{1}{2 t} \int_{0}^{2 t} r^{2}(s) \mathrm{d} s\right) \\
& \times e^{K d t} \cdot e^{\frac{M d}{2}} \int_{0}^{2 t} r^{2}(s) \mathrm{d} s
\end{aligned}
$$

using (83) and $r(s) \leqq K+M r^{2}(s)$ as above. Differentiating the explicit formula (see (85)) for $\mathbf{E} \exp \left(\left(b^{2} / 2\right) \int r^{2}(s) d s\right)$ with respect to $b$ one can estimate the obtained expression for $2 b t \leqq \pi / 10$ as follows:

$$
\mathbf{E}\left(b \int_{0}^{2 t} r^{2}(s) \mathrm{d} s\right) \exp \left(\frac{b^{2}}{2} \int_{0}^{2 t} r^{2}(s) \mathrm{d} s\right) \leqq(\text { const }) \cdot t^{2} \exp \left(\frac{z^{2}}{20 t}\right)
$$

(by (const) we shall denote universal constants, not necessary the same ones). Combining this with (86) and with the conditions on $t$ and $d$, one obtains (84).

Now we can easily prove (44):

$$
P_{0}(x, x) \leqq e^{-t H}(x, x) \leqq \frac{e^{t B(x)}}{4 \pi t} \mathbf{E}_{0, x}^{2 t, x} \exp \left(\frac{1}{2} \int_{0}^{2 t}(B(W(s))-B(x)) \mathrm{d} s\right) .
$$

Choose $t:=1 / B(x)$ and apply the estimate (83) from Lemma 6.1 with $F(w):=$ $B(x+w)-B(x)$ using (11) and (12).

Upper Part of the Spectrum; Proof of (45). To treat the contribution from the upper part of the spectrum of $H$ first we have to present an operator $M_{E, 0}$ with a kernel satisfying (37), and prove (45). 
The first idea is to realize that $\left(I-P_{0}\right) H\left(I-P_{0}\right) \geqq 2 B_{0}$ because of the spectral gap (the spectrum of $H$ has a gap of size at least $2 B_{0}$ above 0 , for details see [CFKS]). On the other hand, for $u \geqq 2 B_{0}$,

$$
e^{-t u} \leqq(\text { const })\left(e^{-t u}-e^{-(t+\beta) u}\right)
$$

with $\beta:=1 /\left(2 B_{0}\right)$, therefore

$$
\left(I-P_{0}\right) e^{-t H}\left(I-P_{0}\right) \leqq(\text { const })\left(e^{-t H}-e^{-(t+\beta) H}\right)
$$

as operators on $L^{2}\left(\mathbf{R}^{2}\right)$. (Note that it is enough to check this inequality on $\operatorname{Ran}(I-$ $\left.P_{0}\right)$, where $H \geqq 2 B_{0}=\beta^{-1}$, since on $\operatorname{Ran}\left(P_{0}\right)$ both sides are 0 .) Extending this inequality to $L^{2}\left(\mathbf{R}^{3}\right)$ and multiplying it with $\exp \left[-t\left(p_{3}^{2}+\frac{E}{2}\right)\right]$ we obtain

$$
\left(I-\Pi_{0}\right) e^{-t\left(H+p_{3}^{2}+\frac{E}{2}\right)}\left(I-\Pi_{0}\right) \leqq(\text { const }) \cdot e^{-t\left(p_{3}^{2}+\frac{E}{2}\right)}\left(e^{-t H}-e^{-(t+\beta) H}\right)
$$

(here we use that if $0 \leqq A \leqq B$, and $C \geqq 0$ commutes with $A$ and $B$, then $A C \leqq$ $B C$ ). Then we use the idea (79) again, namely that

$$
\frac{1}{u+\frac{E}{2}} \leqq(\text { const }) \cdot \int_{0}^{\beta} e^{-t\left(u+\frac{E}{2}\right)} \mathrm{d} t
$$

if $u \geqq \beta^{-1}$.

Therefore, by (91) and (92)

$$
\begin{aligned}
& \left(I-\Pi_{0}\right)\left(H+p_{3}^{2}+\frac{E}{2}\right)^{-1}\left(I-\Pi_{0}\right) \\
& \quad \leqq(\text { const }) \cdot \int_{0}^{\beta} \mathrm{d} t\left(I-\Pi_{0}\right) e^{-t\left(H+p_{3}^{2}+\frac{E}{2}\right)}\left(I-\Pi_{0}\right) \leqq M_{E, 0},
\end{aligned}
$$

where

$$
M_{E, 0}:=(\text { const }) \cdot \int_{0}^{\beta} e^{-t\left(p_{3}^{2}+\frac{E}{2}\right)}\left(e^{-t H}-e^{-(t+\beta) H}\right) \mathrm{d} t,
$$

so (37) is satisfied.

By Proposition 4.1 (and especially by the estimate (58)), it is clear that $M_{E, 0}(\mathbf{x}, \mathbf{y})$ exists for $\mathbf{x} \neq \mathbf{y}$, and

$$
M_{E, 0}(\mathbf{x}, \mathbf{y}) \leqq(\text { const }) \cdot \int_{0}^{\beta} \frac{1}{t^{3 / 2}} \cdot e^{\frac{-(\mathbf{x}-\mathbf{y})^{2}}{8 t}} \Gamma(t, x) \mathrm{d} t<\infty .
$$

Therefore $M_{E, 0}^{2}$ has a kernel even for $\mathbf{x}=\mathbf{y}$, since using the estimate (95),

$$
\begin{aligned}
M_{E, 0}^{2}(\mathbf{x}, \mathbf{x}) & =\int_{\mathbf{R}^{3}} M_{E, 0}(\mathbf{x}, \mathbf{z}) M_{E, 0}(\mathbf{z}, \mathbf{x}) \mathrm{d} \mathbf{z} \\
& \leqq(\text { const }) \cdot \int_{0}^{\beta} \int_{0}^{\beta} \frac{\Gamma(t, x) \Gamma(s, x)}{(s+t)^{3 / 2}} \mathrm{~d} s \mathrm{~d} t<\infty
\end{aligned}
$$

(the existence of $M_{E, 0}^{2}(\mathbf{x}, \mathbf{y})$ for $\mathbf{x} \neq \mathbf{y}$ is even more obvious). 
Calculating $M_{E, 0}^{2}(\mathbf{x}, \mathbf{x})$ from (94) one obtains

$$
\begin{aligned}
M_{E, 0}^{2}(\mathbf{x}, \mathbf{x})= & (\text { const }) \cdot \int_{0}^{\beta} \mathrm{d} t \int_{0}^{\beta} \mathrm{d} s \int_{\mathbf{R}^{3}} \mathrm{~d} \mathbf{y}\left[e^{-t\left(p_{3}^{2}+\frac{E}{2}\right)}\left(e^{-t H}-e^{-(t+\beta) H}\right)\right](\mathbf{x}, \mathbf{y}) \\
& \times\left[e^{-s\left(p_{3}^{2}+\frac{E}{2}\right)}\left(e^{-s H}-e^{-(s+\beta) H}\right)\right](\mathbf{y}, \mathbf{x}) .
\end{aligned}
$$

The main point in this computation was that we wanted to estimate the projected resolvent kernel by the heat kernel (so that we could use the Feynman$\mathrm{Kac}$ formula). On the other hand, the heat kernel is always larger than the ground state projection kernel $P_{0}$, which grows linearly with $B$ (this is why we had to treat its contribution separately), but we need a $B$-independent estimate. Therefore we have to deal with the difference of two heat kernels, so that $P_{0}$ is cancelled.

The next problem is that we will be able to estimate the heat kernel effectively from above (via Feynman-Kac), but obtaining the lower bound is much harder. The best thing we can do is to introduce an approximating Hamiltonian $H_{c}$ with constant magnetic field, use that $e^{-t H_{c}}-e^{-(t+\beta) H_{c}}$ can be exactly calculated, and prove that $e^{-t H}-e^{-t H_{c}}$ is small. This last statement can be proved only for small $t$, this is why the truncation in the limit of integration in (92) was needed (this step shows implicitly that the positive lower bound on $B(x)$ is necessary for the proof). So we anticipate the following Main Lemma:

Main Lemma 6.2. Assume the conditions of Theorem 2.2 and choose a gauge $\mathbf{A}=(A, 0)$ so that $A$ satisfies the conditions (ii) of Proposition 4.1. Fix $x, y \in \mathbf{R}^{2}$ and let $B:=B(x)$. For any $z=\left(z_{1}, z_{2}\right) \in \mathbf{R}^{2}$, define the following divergence free gauge (written as a 1 -form on $\mathbf{R}^{2}$ ):

$$
A^{z}(u):=\frac{B(z)}{2}\left[\left(u_{1}-z_{1}\right) d u_{2}-\left(u_{2}-z_{2}\right) d u_{1}\right]
$$

generating the constant $B(z)$ magnetic field: $\operatorname{rot} A^{z}(u)=B(z)$. Let $H_{c}:=$ $\left(p-A^{x}\right)^{2}-B$ be the operator with the constant $B=B(x)$ magnetic field. Then there exist a real number $\varphi=\varphi(x, y)$ and a constant $C=C(c)$ depending only on $c$ (the constant appearing in (11) in Theorem 2.2), such that for any $t \leqq 1 / B_{0}$ we have

$$
\left|e^{-t H}(x, y)-e^{\imath \varphi} e^{-t H_{c}}(x, y)\right| \leqq \frac{C}{4 \pi t} e^{-\frac{(x-y)^{2}}{8 t}},
$$

or, equivalently,

$$
\begin{aligned}
& \left|\mathbf{E}_{0, x}^{2 t, y}\left(e^{-i \int_{0}^{2 t} A(W(s)) \circ \mathrm{d} W(s)+\frac{1}{2} \int_{0}^{2 t} B(W(s)) \mathrm{d} s}-e^{\imath \varphi} e^{-i \int_{0}^{2 t} A^{x}(W(s)) \mathrm{od} W(s)+B t}\right)\right| \\
& \quad \leqq C \cdot e^{\frac{(\alpha-y)^{2}}{s t}} .
\end{aligned}
$$

Estimating $\left(e^{-(t+\beta) H_{c}}-e^{-t H_{c}}\right)(x, y)$ is easy using the explicit formula (see e.g. [S-1979]):

$$
e^{-t H_{c}}(x, y)=\frac{B e^{B t}}{4 \pi \sinh B t} \exp \left(-B \operatorname{coth}(B t) \frac{(x-y)^{2}}{4}\right)
$$


and its derivative with respect to $t$. Notice that

$$
\left|\left(\frac{\mathrm{d}}{\mathrm{d} \tau} e^{-\tau H_{c}}\right)(x, y)\right| \leqq \frac{\text { const }}{\tau^{2}} e^{-\frac{(x-y)^{2}}{8 \tau}}
$$

independently of $B$, although $e^{-\tau H_{c}}$ itself grows linearly with $B$. Therefore, using the Main Lemma 6.2 for $t \leqq \beta=1 /\left(2 B_{0}\right)$, we have

$$
\begin{aligned}
& \left|\left(e^{-t H}-e^{-(t+\beta) H}\right)(x, y)\right| \\
& \quad \leqq\left[(\text { const }) \int_{t}^{t+\beta} \frac{\mathrm{d} \tau}{\tau^{2}} e^{-\frac{(x-y)^{2}}{8 \tau}}+\frac{C}{4 \pi t} e^{-\frac{(x-y)^{2}}{8 t}}+\frac{C}{4 \pi(t+\beta)} e^{-\frac{(x-y)^{2}}{8(t+\beta)}}\right] .
\end{aligned}
$$

Now we plug this estimate into (97). The dy integration is done explicitly, and then we arrive at the following complicated $\mathrm{d} s$ and $\mathrm{d} t$ integral

$$
\begin{aligned}
(97) \leqq & C^{\prime} \int_{0}^{\beta} \mathrm{d} t \int_{0}^{\beta} \mathrm{d} s \frac{1}{\sqrt{t+s}} \cdot e^{-(s+t) \frac{E}{2}} \\
& \times\left[\int_{t}^{t+\beta} \mathrm{d} \tau \int_{s}^{s+\beta} \mathrm{d} \zeta \frac{1}{\tau \zeta(\tau+\zeta)}+\int_{t}^{t+\beta} \frac{\mathrm{d} \tau}{\tau(\tau+s)}+\int_{t}^{t+\beta} \frac{\mathrm{d} \tau}{\tau(\tau+s+\beta)}+\int_{s}^{s+\beta} \frac{\mathrm{d} \zeta}{\zeta(\zeta+t)}\right. \\
& \left.+\int_{s}^{s+\beta} \frac{\mathrm{d} \zeta}{\zeta(\zeta+t+\beta)}+\frac{1}{t+s}+\frac{2}{t+s+\beta}+\frac{1}{t+s+2 \beta}\right] \\
\leqq & C^{\prime} \int_{0}^{\beta} \mathrm{d} t \int_{0}^{\beta} \mathrm{d} s \frac{1}{\sqrt{s+t}} \cdot e^{-(t+s) \frac{E}{2}} \\
& \times\left[\int_{t}^{t+\beta} \mathrm{d} \tau \int_{s}^{s+\beta} \mathrm{d} \frac{1}{\tau \zeta(\tau+\zeta)}+2 \int_{t}^{t+\beta} \frac{\mathrm{d} \tau}{\tau(\tau+s)}+2 \int_{s}^{s+\beta} \frac{\mathrm{d} \zeta}{\zeta(\zeta+t)}+\frac{4}{t+s}\right], \quad(104)
\end{aligned}
$$

where $C^{\prime}$ depends on the constant $C$ obtained in the Main Lemma 6.2. The righthand side of (104) is monotone increasing in $\beta$ and we need a $\beta=1 /\left(2 B_{0}\right)$ independent estimate for (45), so we can take immediately $\beta=\infty$ :

$$
\begin{aligned}
(97) \leqq & C^{\prime} \int_{0}^{\infty} \mathrm{d} t \int_{0}^{\infty} \mathrm{d} s \frac{1}{\sqrt{t+s}} \cdot e^{-(s+t) \frac{E}{2}} \\
& \times\left[\int_{t}^{\infty} \mathrm{d} \tau \int_{s}^{\infty} \mathrm{d} \zeta \frac{1}{\tau \zeta(\tau+\zeta)}+2 \int_{t}^{\infty} \frac{\mathrm{d} \tau}{\tau(\tau+s)}+2 \int_{s}^{\infty} \frac{\mathrm{d} \zeta}{\zeta(\zeta+t)}+\frac{4}{t+s}\right] \\
\leqq & C^{\prime} \int_{0}^{\infty} \mathrm{d} t \int_{0}^{\infty} \mathrm{d} s \frac{1}{\sqrt{t+s}} e^{-(s+t) \frac{E}{2}} \\
& \times\left[\int_{t}^{\infty} \frac{\mathrm{d} \tau}{\tau^{2}} \log \left(1+\frac{\tau}{S}\right)+\frac{2}{S} \log \left(1+\frac{s}{t}\right)+\frac{2}{t} \log \left(1+\frac{t}{s}\right)+\frac{4}{t+s}\right] \\
\leqq & C^{\prime} \cdot(\text { const }) \int_{0}^{\infty} \mathrm{d} t \int_{0}^{\infty} \mathrm{d} s \frac{1}{\sqrt{t+s}} \cdot e^{-(s+t) \frac{E}{2}}\left[\frac{1}{\sqrt{t s}}+\frac{1}{t+s}\right] \\
\leqq & \frac{C^{\prime} \cdot(\text { const })}{\sqrt{E}} \int_{0}^{\infty} \mathrm{d} T \int_{0}^{\infty} \mathrm{d} S \frac{e^{-(T+S)}}{\sqrt{T S(T+S)}}=\frac{C_{2}}{\sqrt{E}}
\end{aligned}
$$

(using that $\log (1+u) \leqq \sqrt{u}$ ), which proves (45), and so Theorem 2.2. 


\section{Proof of the Main Lemma}

This section contains the essence of the whole proof; we compare the heat kernel of the operator with a nonconstant field with that of the operator with the frozen constant field. We use a localization technique in path space; a similar method has been used in [E-1994(a)], but the present setup is more complicated since we need better estimates. Nevertheless, the intuitive idea of the method outlined in Sect. 2 of [E-1994(a)] might help to understand the present proof.

Introduce the following notations:

$$
\begin{gathered}
F^{z}(w):=B(z+w)-B(z), \\
G^{z}(w):=\left(\int_{0}^{1} t F^{z}(t w) \mathrm{d} t\right)\left(w_{1} \mathrm{~d} w_{2}-w_{2} \mathrm{~d} w_{1}\right),
\end{gathered}
$$

then $G^{z}$ is a 1 -form generating $F^{z}$, i.e. $\mathrm{d} G^{z}(w)=F^{z}(w)$ (we use the canonical identification between 1 -forms $A=A_{1} \mathrm{~d} x_{1}+A_{2} \mathrm{~d} x_{2}$ and vectorfields $A=\left(A_{1}, A_{2}\right)$ without any further comment).

Let

$$
A_{*}^{z}(u):=A(u)-G^{z}(u-z),
$$

then $\mathrm{d} A_{*}^{z}(u)=B(u)-F^{z}(u-z)=B(z)$, so $A_{*}^{z}$ and $A^{z}$ both generate the constant $B(z)$ field. Therefore, there is a function $\varphi^{z}: \mathbf{R}^{2} \rightarrow \mathbf{R}$ such that $A_{*}^{z}=A^{z}+\mathrm{d} \varphi^{z}$. The phase difference $\varphi=\varphi(x, y)$ in the Main Lemma 6.2 will be given as $\varphi:=$ $\varphi^{x}(x)-\varphi^{x}(y)$.

We will not give the exact value of $C=C(c)$, but it is explicitly computable from the proof below. Also, we will use the same letter $C$ for various positive constants depending only on $c$.

First we eliminate some extreme cases.

Case 1 (short time). If $B t \leqq 1$ (recall that $B:=B(x)$ ), then by the roughest estimate

$$
\begin{aligned}
\text { LHS of }(100) & \leqq e^{B t}+\mathbf{E}_{0, x}^{2 t, y} e^{\frac{1}{2} \int_{0}^{2 t} B(W(s)) \mathrm{d} s} \leqq e^{B t}\left(1+\mathbf{E}_{0, x}^{2 t, y} e^{\frac{1}{2} \int_{0}^{2 t} F^{\mathrm{r}}(W(s)-x) \mathrm{d} s}\right) \\
& \leqq e^{B t}\left(1+C^{(0)} \cdot e^{\frac{(x-y)^{2}}{40 t}}\right) \leqq C \cdot e^{\frac{(x-y)^{2}}{8 t}},
\end{aligned}
$$

using Lemma 6.1.

Case 2 (large distance). If $(x-y)^{2} \geqq 16 B t^{2}$, then $B t \leqq(x-y)^{2} /(16 t)$, so one can use the same rough estimate (109) as above to obtain

$$
\text { LHS of }(100) \leqq e^{\frac{(x-y)^{2}}{10 t}}\left(1+C^{(0)} e^{\frac{(x-y)^{2}}{40 t}}\right) \leqq C \cdot e^{\frac{(x-y)^{2}}{8 t}}
$$

So from now on we can assume that $B t \geqq 1,(x-y)^{2} \leqq 16 B t^{2}$ and we have to bound the expression

$$
I:=\left|\mathbf{E}\left(e^{-i \int A \circ \mathrm{od} W+\frac{1}{2} \int B}-e^{l \varphi} e^{-l \int A^{\prime} \mathrm{od} W+B t}\right)\right|
$$


from above (for brevity, we use a straightforward shorthand notation when it makes no confusion).

7.1. A Sequence of Stopping Times. Let $\varepsilon:=2 t /([B t]+1)$ (here [] denotes the integer part) and we define a sequence of stopping times $\tau_{\iota}$ inductively as follows. Let $\tau_{0}:=0, x_{j}:=W\left(\tau_{j}\right)$ and for $j \geqq 0$ let

$$
\tau_{j+1}:=\varepsilon\left[\frac{s_{j+1}}{\varepsilon}+1\right]
$$

where

$$
s_{j+1}:=\inf \left\{r: \tau_{j}<r \leqq 2 t \text { and }\left|\int_{\tau_{j}}^{\tau} A^{x_{J}}(W(s)) \mathrm{d} W(s)\right|=\frac{\pi}{2}\right\},
$$

which is also a stopping time (if there is no such $r$ then we stop defining the sequence $\tau_{j}$ ). The crucial idea is that $s_{j+1}$ is the first time when the flux of the frozen constant magnetic field $B\left(x_{j}\right)$ between the Brownian curve starting at time $\tau_{j}$ from the point $x_{j}$ and the corresponding chord reaches $\pi / 2$ in absolute value. After that, we look for the next stopping time with the same property, but for technical reasons we have to discretize the set of the starting times, this is why we introduce the $\tau$ 's.

Let $\tau_{n(W)}$ be the last stopping time defined above $(n(W) \leqq[t B]+1=2 t / \varepsilon$ is an integer valued random variable). Define $\mathscr{H}_{n}:=\{W: n(W)=n\}$, then clearly $\mathbf{P}\left(\cup_{n} \mathscr{H}_{n}\right)=1$, and define the $j^{\text {th }}$ reflection $T_{j}: \mathscr{H}_{n} \rightarrow \mathscr{H}_{n}(0 \leqq j \leqq n-1)$ in the following way. It will affect only the $\left\{W(s): \tau_{j} \leqq s \leqq s_{j+1}\right\}$ part of the Brownian bridge, so let $\left[T_{j}(W)\right](s):=W(s)$ for $s<\tau_{j}$ or $s>s_{j+1}$. For $\tau_{j} \leqq s \leqq s_{j+1}$, let $\left[T_{j}(W)\right](s)$ be the geometric reflection of $W(s)$ onto the segment $\left[W\left(\tau_{j}\right), W\left(s_{j+1}\right)\right]$. By the strong Markov property, $T_{j}$ preserves the probability measure and the sequence of stopping times $\tau_{j}$, and $T_{j}$ is an involution. These last two statements follow from the crucial relation:

$$
\int_{\tau_{j}}^{\tau} A^{x_{j}}(W(s)) \mathrm{d} W(s)=-\int_{\tau_{j}}^{\tau} A^{x_{j}}(\bar{W}(s)) \mathrm{d} \bar{W}(s)
$$

for any $\tau_{j} \leqq r \leqq s_{j+1}$, where $\bar{W}:=T_{j}(W)$ for simplicity.

Define the following stochastic integrals for $0 \leqq j \leqq n=n(W)$ for paths $W$ belonging to $\mathscr{H}_{n}$ :

$$
\begin{aligned}
& N_{j}(W)=N_{j}:=-i \int_{\tau_{j}}^{\tau_{j}+1} G^{W\left(\tau_{j}\right)}\left(W(s)-W\left(\tau_{j}\right)\right) \circ \mathrm{d} W(s) \\
&+\frac{1}{2} \int_{\tau_{j}}^{\tau_{j+1}} F^{W\left(\tau_{j}\right)}\left(W(s)-W\left(\tau_{j}\right)\right) \mathrm{d} s, \\
& M_{j}(W)=M_{j}:=-\int_{\tau_{j}}^{\tau_{j+1}} A_{*}^{W\left(\tau_{j}\right)}(W(s)) \circ \mathrm{d} W(s), \\
& L_{j}(W)=L_{j}:=\frac{1}{2} B\left(W\left(\tau_{j}\right)\right)\left(\tau_{j+1}-\tau_{j}\right),
\end{aligned}
$$


where $\tau_{n+1}:=2 t$ (it might be that $\tau_{n}=2 t$, then $N_{n}=M_{n}=L_{n}=0$ ). Let $\bar{N}_{j}:=$ $N_{j}\left(T_{j} W\right)$ and $\bar{M}_{j}:=M_{j}\left(T_{j} W\right)$ be the same quantities for the $T_{j}$-reflected path. Then, for $0 \leqq j \leqq n-1$ we have $\left|M_{j}-\bar{M}_{j}\right|=\pi$ by the careful definition of the stopping times, since

$$
\begin{aligned}
\left|M_{j}-\bar{M}_{j}\right| & =\left|\int_{\tau_{j}}^{\tau_{j+1}} A_{*}^{W\left(\tau_{j}\right)}(W(s)) \circ \mathrm{d} W(s)-\int_{\tau_{j}}^{\tau_{j+1}} A_{*}^{W\left(\tau_{j}\right)}(\bar{W}(s)) \circ \mathrm{d} \bar{W}(s)\right| \\
& =\left|\int_{\tau_{j}}^{s_{j+1}} A^{W\left(\tau_{j}\right)}(W(s)) \circ \mathrm{d} W(s)-\int_{\tau_{j}}^{s_{j+1}} A^{W\left(\tau_{j}\right)}(\bar{W}(s)) \circ \mathrm{d} \bar{W}(s)\right|=\pi,
\end{aligned}
$$

using first the fundamental theorem of calculus for the Stratonovich integral, then the fact that $A^{z}$ is divergence free, so its Ito and Stratonovich integrals are the same, and finally the relation (113) and the definition of $s_{j+1}$.

We shall decompose the quantity $I$ to be estimated (see (111)) according to the disjoint events $\mathscr{H}_{n}$ :

$$
I=\left|\sum_{n=0}^{2 t / \varepsilon} I_{n}\right|
$$

with

$$
I_{n}:=\mathbf{E}_{0, x}^{2 t, y} \chi\left(\mathscr{H}_{n}\right)\left(e^{-i \int A \circ \mathrm{d} W+\frac{1}{2} \int B}-e^{i \varphi} e^{-l \int A^{x} \circ \mathrm{d} W+B t}\right) .
$$

The $n=0$ case must be treated separately; on this event the contribution from both terms in (111) is proportional to $B$, but they will cancel each other. In operator language this corresponds to the ground states; we know that the heat kernel contains the ground state projection, which is proportional to $B$, but we need a $B$-independent estimate. On the other hand, we wanted to estimate the heat kernel only on the subspace orthogonal to the ground states, which allowed us to subtract another heat kernel (namely that of the constant magnetic field) having more or less the same ground state projection as $H$. This was the essence of the calculation in Sect. 6 .

7.2. Estimating $I_{0}$ (No Reflection). Using the notations above, we have

$$
\left|I_{0}\right| \leqq e^{B t} \mathbf{E}_{0, x}^{2 t, y}\left(\chi\left(\mathscr{H}_{0}\right)\left|e^{N_{0}}-1\right|\right) .
$$

We have to treat the largely deviating bridges separately. Let $R:=8 t \sqrt{B}$ and let

$$
E:=\left\{W(s): \sup _{0 \leqq s \leqq 2 t}|W(s)-x| \leqq R\right\}
$$

be a measurable subset of the path space. By standard large deviation estimate (see e.g. [S-1984]) for the complement of $E$ we have

$$
\mathbf{P}_{0, x}^{2 t, y}\left(E^{c}\right) \leqq 4 e^{-\frac{R^{2}}{16 t}} \leqq 4 e^{-4 B t},
$$


and on the subset $E^{c}$, the left-hand side of (120) can be easily estimated by

$$
\begin{aligned}
e^{B t} & \mathbf{E}_{0, x}^{2 t, y}\left(\chi\left(E^{c}\right) \chi\left(\mathscr{H}_{0}\right)\left|e^{N_{0}}-1\right|\right) \\
& \leqq e^{B t}\left(\mathbf{P}_{0, x}^{2 t, y}\left(E^{c}\right)+\mathbf{E}_{0, x}^{2 t, y} \chi\left(E^{c}\right) e^{\frac{1}{2} \int_{0}^{2 t} F^{x}(W(s)-x) \mathrm{d} s}\right) \\
& \leqq e^{B t}\left(\mathbf{P}_{0, x}^{2 t, y}\left(E^{c}\right)+\left(\mathbf{P}_{0, x}^{2 t, y}\left(E^{c}\right) \cdot \mathbf{E}_{0,0}^{2 t, y-x} e^{\int_{0}^{2 t} F^{x}(W(s)) \mathrm{d} s}\right)^{1 / 2}\right) \\
& \leqq C \cdot e^{\frac{(x-y)^{2}}{8 t}},
\end{aligned}
$$

using Lemma 6.1 again (now use it with $2 F^{x}$ instead of $F^{x}$, so the constant $C$ obtained here is essentially $C^{(0)}(2 c)$ with the notations of Lemma 6.1).

On the subset $E$, by the general estimate $\left|e^{i X+Y}-1\right| \leqq|X|+|Y| e^{|Y|}$ for real numbers $X$ and $Y$, we have

$$
\begin{gathered}
e^{B t} \mathbf{E}_{0, x}^{2 t, y}\left(\chi\left(E \cap \mathscr{H}_{0}\right)\left|e^{N_{0}}-1\right|\right) \\
\leqq e^{B t} \mathbf{E}_{0, x}^{2 t, y}\left(\chi ( E \cap \mathscr { H } _ { 0 } ) \left(\left|\int_{0}^{2 t} G^{x}(W(s)-x) \mathrm{d} W(s)\right|+\frac{1}{2}\left|\int_{0}^{2 t} \operatorname{div} G^{x}(W(s)-x) \mathrm{d} s\right|\right.\right. \\
\left.\left.\quad+\frac{1}{2} \int_{0}^{2 t}\left|F^{x}(W(s)-x)\right| \mathrm{d} s \cdot e^{\frac{1}{2} \int_{0}^{2 t} F^{x}(W(s)-x) \mathrm{d} s}\right)\right)
\end{gathered}
$$

Notice that we have replaced the Stratonovich integral by the Ito integral plus the divergence term. The reason is that the Ito integral is a martingale so the calculations become easier.

We estimate each term in (124) separately. For the last term use that

$$
\left|F^{x}(W(s)-x)\right| \leqq c d(x)|W(s)-x| \leqq c d(x) R
$$

on the event $E$ to obtain that

$$
\text { Last term on the RHS of }(124) \leqq e^{B t} \mathbf{P}_{0, x}^{2 t, y}\left(\mathscr{H}_{0}\right) c d(x) R t \cdot e^{c d(x) R t} .
$$

For estimating the probability of $\mathscr{H}_{0}$, we recall Lemma 4.1 from [E-1994(a)] (in a simplified form)

Lemma 7.1. For any $\bar{x}, \bar{y} \in \mathbf{R}^{2}$ let

$$
\bar{\xi}(s):=\frac{\bar{B}}{2} \int_{0}^{s}\left(W_{1}(u)-\bar{x}_{1}\right) \mathrm{d} W_{2}(u)-\left(W_{2}(u)-\bar{x}_{2}\right) \mathrm{d} W_{1}(u)
$$

be the random flux process of the two dimensional Brownian bridge under the constraints $W(0)=\bar{x}, W(2 \bar{t})=\bar{y}$ for the constant magnetic field $\bar{B}$. Assume that

$$
\bar{B} \bar{t} \geqq \bar{c}
$$


for some positive $\bar{c}$, then there exists a constant $\bar{C}=\bar{C}(\bar{c})$ depending only on $\bar{c}$ such that

$$
\mathbf{P}_{0, \bar{\gamma}}^{2 \bar{i} \bar{y}}\left(\sup _{0 \leqq s \leqq 2 \bar{t}}|\bar{\zeta}(s)|<\frac{\pi}{2}\right) \leqq \bar{C}(1+\bar{B} \bar{t}) e^{-\bar{B} \bar{i}}
$$

By the definition of $A^{x}$ (see (98)) and the sequence of stopping times $\tau_{j}$, we have

$$
\mathbf{P}_{0, .1}^{2 t, v}\left(\mathscr{H}_{0}\right)=\mathbf{P}_{0 . x}^{2 t, v}\left(\sup _{0 \leqq s \leqq 2 t}\left|\int_{0}^{s} A^{\prime}(W(u)) \mathrm{d} W(u)\right|<\frac{\pi}{2}\right) \leqq(\text { const })(1+B t) e^{-B t}
$$

after applying Lemma 7.1 (recall that $B t \geqq 1$ ). Plugging (130) and the value $R:=8 t \sqrt{B}$ into (126), we have

$$
\text { Last term in }(124) \leqq(\text { const }) B t \cdot \sqrt{B} t^{2} c d(x) \cdot e^{8 \sqrt{B} t^{2} c d(r)} \leqq C
$$

by (12) and $t \leqq 1 / B_{0}$.

The estimate of the divergence term in (124) is similar. By the definition of $G^{x}$ (see (107)) clearly

$$
\left|\operatorname{div} G^{r}(w)\right| \leqq|w| \cdot \sup _{[0, w]}\left|\nabla F^{r}\right|=|w| \cdot \sup _{u \in[r, r+w]}|\nabla B(u)| \leqq c|w| \cdot \sup _{u \in[x, r+w]} d(u),
$$

since the function $c d(u)$ clearly dominates $|\nabla B(u)|$ by $(11)\left([x, x+w] \subset \mathbf{R}^{2}\right.$ denotes the segment joining $x$ and $x+w)$. For $|u-x| \leqq R$ we have

$$
|B(x)-B(u)| \leqq c B_{0}^{3 / 2} \sqrt{\frac{B_{0}}{B(x)}} R \leqq 8 c B_{0},
$$

especially $B(u) \geqq B(x)-8 c B_{0}$, which implies, in particular, that $B(u) \geqq B(x) /(1+$ $8 c$ ) (recall that $\left.B(u) \geqq B_{0}\right)$, therefore

$$
|u-x| \leqq R \Rightarrow B(u) \geqq C B(x) \text { and } d(u) \leqq C d(x)
$$

(recall that $C$ denotes different positive constants depending only on $c$ ). Therefore for paths in $E$,

$$
\left|\operatorname{div} G^{x}(W(s)-x)\right| \leqq|W(s)-x| \cdot \sup _{u:|u-1| \leqq R} d(u) \leqq C R d(x),
$$

which allows us to estimate the divergence term in (124) as follows

$$
\text { Second term on the RHS of }(124) \leqq C e^{B t} t R d(x) \mathbf{P}_{0, x}^{2 t, y}\left(\mathscr{H}_{0}\right) \leqq C \text {. }
$$

Finally, we have to estimate the first term on the right-hand side of (124). This is much harder since one cannot plug an upper estimate on the integrand into a stochastic integral. First, we have to estimate the stochastic integral by an ordinary integral using the Kolmogorov inequality for martingales. This is the content of Lemma 4.3 in [E-1994(a)] which we recall here for convenience: 
Lemma 7.2. Let $W$ be the Brownian bridge in $\mathbf{R}^{2}$ with $W(0)=0$ and $W(2 \theta)=z$. Then for any function $H: \mathbf{R}^{2} \rightarrow \mathbf{R}^{2}$ with at most polynomial growth and $\mu \geqq 1$ integer,

$$
\mathbf{E}\left(\int_{0}^{2 \theta} H(W(s)) \mathrm{d} W(s)\right)^{2 \mu} \leqq(\text { const })^{\mu} \mu^{4 \mu / 3}\left(z^{2 \mu}+\theta^{\mu}\right) \cdot \mathbf{E}^{1 / 4} \frac{1}{2 \theta} \int_{0}^{2 \theta}|H(W(s))|^{8 \mu} \mathrm{d} s
$$

Before applying this lemma we have to separate the $\chi\left(\mathscr{H}_{0} \cap E\right)$ factor since on a restricted set the stochastic integral is not a martingale.

Let $\mu:=[B t]$ (integer part) and use Hölder's inequality

$$
\begin{aligned}
& e^{B t} \mathbf{E}_{0, x}^{2 t, y}\left(\chi\left(\mathscr{H}_{0} \cap E\right)\left|\int_{0}^{2 t} G^{x}(W(s)-x) \mathrm{d} W(s)\right|\right) \\
& \leqq e^{B t}\left(\mathbf{P}_{0, x}^{2 t, y}\left(\mathscr{H}_{0}\right)\right)^{1-\frac{1}{2 \mu}}\left(\mathbf{E}_{0, x}^{2 t, y}\left(\int_{0}^{2 t} G^{x}(W(s)-x) \mathrm{d} W(s)\right)^{2 \mu}\right)^{\frac{1}{2 \mu}}
\end{aligned}
$$

For the probability of $\mathscr{H}_{0}$ we use the same estimate as before; notice that this factor still cancels $e^{B t}$ essentially, since

$$
\left(\mathbf{P}_{0, x}^{2 t, y}\left(\mathscr{H}_{0}\right)\right)^{1-\frac{1}{2 \mu}} \leqq\left((\text { const })(1+B t) e^{-B t}\right)^{1-\frac{1}{2 \mu}} \leqq(\text { const })(1+B t) e^{-B t}
$$

since $B t \leqq 2 \mu$ by $B t \geqq 1$. For the other term in (138), we use Lemma 7.2 to obtain RHS of (138)

$$
\begin{aligned}
& \leqq(\text { const })(1+B t) \mu^{2 / 3}\left((x-y)^{2 \mu}+t^{\mu}\right)^{\frac{1}{2 \mu}}\left(\mathbf{E}_{0, x}^{2 t, y} \frac{1}{2 t} \int_{0}^{2 t}\left|G^{x}(W(s)-x)\right|^{8 \mu} \mathrm{d} s\right)^{\frac{1}{8 \mu}} \\
& \leqq(\text { const }) c d(x) \sqrt{t}(B t)^{5 / 3}\left(1+\frac{(x-y)^{2}}{t}\right)^{1 / 2}\left(\mathbf{E}_{0, x}^{2 t, y} \frac{1}{2 t} \int_{0}^{2 t}|(W(s)-x)|^{16 \mu} \mathrm{d} s\right)^{\frac{1}{8 \mu}},
\end{aligned}
$$

where, in addition to some arithmetic estimates, we have used that

$$
\left|G^{x}(w)\right| \leqq|w| \cdot \sup _{u \in[0, w]}\left|F^{x}(u)\right| \leqq|w| \cdot \sup _{u \in[0, w]}|B(u+x)-B(x)| \leqq c d(x)|w|^{2}
$$

based upon (107).

Estimating the expectation in (140) is standard; one uses the following representation for the Brownian bridge:

$$
W(s)=x+(y-x) \cdot \frac{s}{2 t}+\sqrt{2 t} b\left(\frac{s}{2 t}\right)
$$

where $b(u)$ is the standard two-dimensional Brownian loop under the constraints $b(0)=b(1)=0$. We denote by $\mathbf{E}_{b}$ the expectation with respect to the measure 
of $b(u)$. Therefore

$$
\begin{aligned}
& \mathbf{E}_{0, x}^{2 t, y} \frac{1}{2 t} \int_{0}^{2 t}|W(s)-x|^{16 \mu} \mathrm{d} s \\
& \quad \leqq(\text { const })^{\mu}\left(\frac{1}{2 t} \int_{0}^{2 t}\left(|x-y| \cdot \frac{s}{2 t}\right)^{16 \mu} \mathrm{d} s+\mathbf{E}_{b} \frac{1}{2 t} \int_{0}^{2 t}(2 t)^{8 \mu}\left|b\left(\frac{s}{2 t}\right)\right|^{16 \mu} \mathrm{d} s\right) \\
& \quad \leqq(\text { const })^{\mu}\left(|x-y|^{16 \mu}+(2 t)^{8 \mu}(8 \mu) !\right),
\end{aligned}
$$

using the explicit formula for the moments of $b(u)$ :

$$
\mathbf{E}_{b}|b(u)|^{2 m}=(2 m-1) ! ! \cdot(2 u(1-u))^{m}
$$

for any positive integer $m$.

Plugging (143) and $\mu \leqq B t$ into (140), and using Stirling's formula to estimate the factorial we get

$$
\begin{aligned}
\text { RHS of }(138) & \leqq(\text { const }) c d(x) \sqrt{t}(B t)^{5 / 3}\left(1+\frac{(x-y)^{2}}{t}\right)^{1 / 2}\left((x-y)^{2}+B t^{2}\right) \\
& \leqq(\text { const }) c d(x)(B t)^{8 / 3} t^{3 / 2} \cdot e^{\frac{(x-y)^{2}}{8 t}} \leqq C e^{\frac{(x-y)^{2}}{8 t}}
\end{aligned}
$$

which finishes the estimate of $I_{0}$.

\subsection{Estimating $I_{n}$ for $n \geqq 1$ Using Reflections}

Decomposition of the path. We first note that for $n \geqq 1$ the contribution to (119) from the operator $H^{c}$ (with frozen constant field) is zero, since

$$
\begin{aligned}
& \mathbf{E}\left(\chi\left(\mathscr{H}_{n}\right) e^{-i \int_{0}^{2 t} A^{x}(W(s)) \circ \mathrm{d} W(s)}\right) \\
& \quad=\frac{1}{2} \mathbf{E}\left(\chi\left(\mathscr{H}_{n}\right)\left(e^{-i \int_{0}^{2 t} A^{x}(W(s)) \circ \mathrm{d} W(s)}+e^{-\imath \int_{0}^{2 t} A^{x}(\bar{W}(s)) \circ \mathrm{d} \bar{W}(s)}\right)\right)=0
\end{aligned}
$$

(where $\bar{W}:=T_{0} W$ ), because the difference of the phase factors is exactly $\pi$ (see (117) with $j=0$ ). We use the shorthand notation $\mathbf{E}=\mathbf{E}_{0, x}^{2 t, y}$ and similarly $\mathbf{P}=\mathbf{P}_{0, x}^{2 t, y}$. Therefore, only

$$
\mathbf{E}\left[\left(1-\chi\left(\mathscr{H}_{0}\right)\right) e^{-i \int_{0}^{2 t} A(W(s)) \mathrm{od} W(s)+\frac{1}{2} \int_{0}^{2 t} B(W(s)) \mathrm{d} s}\right]=\sum_{n=1}^{2 t / \varepsilon} \mathbf{E}\left(\chi\left(\mathscr{H}_{n}\right) \prod_{j=0}^{n} e^{N_{j}+i M_{\jmath}+L_{\jmath}}\right)
$$

remains to be estimated. 
The basic idea is that on the set $\mathscr{H}_{n}$ we consider all the $2^{n}$ paths of the form $T^{\underline{\sigma}} W$ together, where $\underline{\sigma} \in\{0,1\}^{n}$ and $T^{\underline{\sigma}}=T_{0}^{\sigma_{0}} T_{1}^{\sigma_{1}} \ldots T_{n-1}^{\sigma_{n-1}}$. Therefore

$$
\begin{array}{r}
\sum_{n \geqq 1} I_{n}=\sum_{n=1}^{2 t / \varepsilon} \frac{1}{2^{n}} \mathbf{E}\left(\chi\left(\mathscr{H}_{n}\right) \sum_{\underline{\sigma} \in\{0,1\}^{n}} \prod_{J=0}^{n-1}\left(e^{l M_{j}\left(T^{\underline{\sigma}} W\right)+N_{J}\left(T^{-} W\right)}\right)\right. \\
\left.\times e^{i M_{n}\left(T^{\underline{\sigma}} W\right)+N_{n}\left(T^{-} W\right)} \prod_{j=0}^{n} e^{L_{J}\left(T^{\underline{\sigma}} W\right)}\right) .
\end{array}
$$

Clearly, $M_{n}, N_{n}$ and $L_{n}$ do not depend on $\underline{\sigma}$, and

$$
\sum_{\underline{\sigma} \in\{0,1\}^{n}} \prod_{j=0}^{n-1}\left(e^{i M_{j}\left(T^{\underline{\sigma}} W\right)+N_{j}\left(T^{\underline{\sigma}} W\right)}\right)= \pm \prod_{j=0}^{n-1} e^{i M_{j}(W)} \prod_{j=0}^{n-1}\left(e^{N_{j}}-e^{\bar{N}_{j}}\right)
$$

using (117). Putting (147), (148) and (149) together, (100) will follow from

$$
\sum_{n=1}^{2 t / \varepsilon} \frac{1}{2^{n}} \mathbf{E}\left(\chi\left(\mathscr{H}_{n} \cap \tilde{E}\right) \prod_{j=0}^{n-1}\left(\left|e^{N_{J}}-e^{\bar{N}_{\jmath}}\right| e^{L_{l}}\right)\left|e^{N_{n}}\right| e^{L_{n}}\right) \leqq C \cdot e^{\frac{(x-y)^{2}}{8 t}},
$$

and from

$$
\sum_{n=1}^{2 t / \varepsilon} \frac{1}{2^{n}} \mathbf{E}\left(\chi\left(\mathscr{H}_{n} \cap \tilde{E}^{c}\right) \prod_{j=0}^{n-1}\left(\left|e^{N_{j}}-e^{\bar{N}_{j}}\right| e^{L_{j}}\right)\left|e^{N_{n}}\right| e^{L_{n}}\right) \leqq C \cdot e^{\frac{(x-y)^{2}}{8 t}},
$$

where

$$
\tilde{E}:=\left\{W(s): \forall \underline{\sigma} \in\{0,1\}^{n(W)} \sup _{0 \leqq s \leqq 2 t}\left|T^{\underline{\sigma}} W(s)-x\right| \leqq R\right\} .
$$

The left-hand side of (151) is estimated very crudely as follows (using that $\tilde{E}$ is invariant under the reflections)

$$
\begin{aligned}
\text { LHS of }(151) & \leqq \sum_{n=1}^{2 t / \varepsilon} \frac{1}{2^{n}} \mathbf{E}\left(\chi\left(\mathscr{H}_{n} \cap \tilde{E}^{c}\right) \prod_{j=0}^{n-1}\left(\left|e^{N_{j}}\right|+\left|e^{\bar{N}_{j}}\right|\right) e^{L_{\jmath}}\left|e^{N_{n}}\right| e^{L_{n}}\right) \\
& \leqq \mathbf{E}\left(\chi\left(E^{c}\right) e^{\frac{1}{2} \int_{0}^{2 t} B(W(s)) \mathrm{d} s}\right) \\
& \leqq e^{B t} \mathbf{P}^{1 / 2}\left(\tilde{E}^{c}\right) \cdot \mathbf{E}^{1 / 2} e^{\int_{0}^{2 t} F^{x}(W(s)-x) \mathrm{d} s} .
\end{aligned}
$$

Now use Lemma 6.1 and that

$$
\mathbf{P}\left(\tilde{E}^{c}\right) \leqq 2^{[B t]+1} \cdot \mathbf{P}\left(E^{c}\right) \leqq(\text { const }) e^{-3 B t}
$$

(since $n(W) \leqq[B t]+1$ for each path) to obtain (151).

For the proof of (150), we are going to split the path $W:[0,2 t] \rightarrow \mathbf{R}^{2}$ into pieces according to the stopping times $\tau_{j}=\varepsilon k_{j}$ (integer $k_{j}$ is defined as $\tau_{j} / \varepsilon$ ). First, we split the event $\mathscr{H}_{n} \cap \tilde{E}$ by defining

$$
E_{j}:=\left\{W(s): n(W)>j \text { and for } \sigma \in\{0,1\} \sup _{\varepsilon k_{J} \leqq s \leqq \varepsilon k_{j+1}}\left|T_{j}^{\sigma} W(s)-x\right| \leqq R\right\}
$$


with the remark that if $\sup |\xi(s)|<\pi / 2$ for $\varepsilon k_{j} \leqq s \leqq \varepsilon k_{j+1}$ (i.e. $\varepsilon k_{j+1}=2 t$ and there is no reflection $T_{j}$ ), then only $\sigma=0$ should be considered in the definition of $E_{J}$. These events clearly depend only on the corresponding part of the path $W(s)$, and

$$
\bigcap_{0 \leqq j \leqq n}\left(E_{J} \cap \mathscr{H}_{n}\right)=\tilde{E} \cap \mathscr{H}_{n} .
$$

Furthermore, let $x_{j}:=W\left(\tau_{j}\right)=W\left(\varepsilon k_{j}\right)$, and let

$$
\xi_{j}(s):=\int_{\varepsilon k_{l}}^{s} A^{x_{j}}(W(u)) \mathrm{d} W(u)
$$

be the flux process starting at $\tau_{j}=\varepsilon k_{j}$ from the point $x_{j}$. We decompose the path (and the corresponding measure) at times $\tau$, using the strong Markov property of the Brownian bridge to get the following formula $(\delta:=\varepsilon / 2)$ :

$$
\begin{aligned}
& \text { LHS of }(150)=\sum_{n=1}^{2 t / \varepsilon} \frac{1}{2^{n}} \cdot(4 \pi t) \cdot e^{\frac{(x-y)^{2}}{4 t}} \int_{\left|x-x_{j}\right| \leqq R} \mathrm{~d} x_{1} \mathrm{~d} x_{2} \ldots \mathrm{d} x_{n} \\
& \qquad \sum_{0<k_{1}<\cdots<k_{n} \leqq 2 t / \varepsilon}\left(\prod_{j=0}^{n-1} \frac{\exp \left(-\frac{\left(x_{j}-1-x_{\jmath}\right)^{2}}{2 \varepsilon\left(k_{j+1}-k_{l}\right)}\right)}{\left(2 \pi \varepsilon\left(k_{j+1}-k_{j}\right)\right)}\right) \cdot \frac{\exp \left(-\frac{\left(y-x_{n}\right)^{2}}{2\left(2 t-\varepsilon k_{n}\right)}\right)}{\left(2 \pi\left(2 t-\varepsilon k_{n}\right)\right)} \\
& \quad \times \prod_{j=0}^{n-1}\left(e ^ { \delta ( k _ { j + 1 } - k _ { j } ) B ( x _ { j } ) } \mathbf { E } _ { \varepsilon k _ { j } , x _ { j } } ^ { \varepsilon k _ { j } , x _ { j + 1 } } \left\{\chi\left(E_{J}\right) \mid e^{N_{J}}-e^{\bar{N}_{j} \mid}\right.\right. \\
& \left.\left.\quad \times \chi\left(\sup _{\varepsilon k_{l} \leqq s \leqq \varepsilon\left(k_{j+1}-1\right)}\left|\xi_{j}(s)\right|<\frac{\pi}{2} \leqq \sup _{\varepsilon k_{j} \leqq s \leqq \varepsilon k_{j+1}}\left|\xi_{j}(s)\right|\right)\right\}\right) \\
& \quad \times\left(e^{\left(t-\delta k_{n}\right) B\left(x_{n}\right)} \mathbf{E}_{\varepsilon k_{n}, x_{n}}^{2 t, y}\left\{\chi\left(E_{n}\right) \cdot\left|e^{N_{n}}\right| \cdot \chi\left(\sup _{\varepsilon k_{n} \leqq s \leqq 2 t}\left|\xi_{n}(s)\right|<\frac{\pi}{2}\right)\right\},\right.
\end{aligned}
$$

where, in the case of $\tau_{n}=\varepsilon k_{n}=2 t$, the exponential factor containing $2 t-\varepsilon k_{n}$ in the denominator is considered 1 .

Local estimates. The following lemma contains the necessary local estimates for various factors of (157):

Lemma 7.3. The following upper bounds hold:

$$
\text { Last line of }(157) \leqq C B t \text {, }
$$

and for any $j$

$$
\text { The } j^{\text {th }} \text { factor in the third and fourth line of }(157) \leqq \frac{C}{(B t)^{2}} \text {. }
$$


Proof. Estimating the last line of (157) is easy. If $2 t=\varepsilon k_{n}$ then it is simply 1, so we can assume that $2 t-\varepsilon k_{n} \geqq \varepsilon$ (recall that $2 t / \varepsilon$ is an integer). Use that on the event $E_{n}$ we have (for $\varepsilon k_{n} \leqq s \leqq 2 t$ )

$$
\left|F^{x_{n}}\left(W(s)-x_{n}\right)\right|=\left|B(W(s))-B\left(x_{n}\right)\right| \leqq c d\left(x_{n}\right)\left|W(s)-x_{n}\right| \leqq C d(x) R
$$

by (134) and $\left|W(s)-x_{n}\right| \leqq|W(s)-x|+\left|x-x_{n}\right| \leqq 2 R$. Now use Lemma 7.1 with $\vec{B}:=B\left(x_{n}\right)$ and

$$
2 \bar{t}:=2 t-k_{n} \varepsilon \geqq \varepsilon \geqq 1 / B \geqq C / B\left(x_{n}\right)=C / \bar{B}
$$

(by (134)) to estimate the probability in the last line of (157) and combine it with (160) and the definition of $N_{n}$ to obtain

Last line of $(157) \leqq e^{\left(t-\delta k_{n}\right) B\left(x_{n}\right)} e^{C d(x) R t} C\left(1+B\left(x_{n}\right)\left(t-k_{n} \delta\right)\right) e^{-\left(t-\delta k_{n}\right) B\left(x_{n}\right)}$

$$
\leqq C B t
$$

(in the last estimate we used again (134)).

To estimate the third and fourth line of (157), we use Hölder's inequality for each fixed $j$ with exponents $P$ and $P /(P-1)$, where $P:=2\left[t B\left(x_{j}\right)\right]+2$ (depending on $j$ ), and we omit a part of the conditions on $\xi_{j}(s)$. Therefore

$$
\begin{aligned}
& \mathbf{E}_{\varepsilon k_{j}, x j}^{\varepsilon k_{j+1}, x_{J+1}}\left\{\chi\left(E_{J}\right)\left|e^{N_{J}}-e^{\bar{N}_{j}}\right| \cdot \chi\left(\sup _{\varepsilon k_{j} \leqq s \leqq \varepsilon\left(k_{J+1}-1\right)}\left|\xi_{j}(s)\right|<\frac{\pi}{2} \leqq \sup _{\varepsilon k_{J} \leqq s \leqq \varepsilon k_{j+1}}\left|\xi_{j}(s)\right|\right)\right\} \\
& \leqq\left\{\mathbf{E}_{\varepsilon k_{j}, x_{j}}^{\varepsilon k_{j}, x_{j+1}} \chi\left(E_{j}\right) \chi\left(T_{j} \text { exists }\right)\left|e^{N_{J}}-e^{\bar{N}_{J}}\right|^{P}\right\}^{1 / P} \\
& \quad \times\left\{\mathbf{P}_{\varepsilon k_{j}, x_{j}}^{\varepsilon k_{j+1}, x_{j+1}}\left(\sup _{\varepsilon k_{J} \leqq s \leqq \varepsilon\left(k_{J+1}-1\right)}\left|\xi_{j}(s)\right|<\frac{\pi}{2}\right)\right\}^{1-1 / P}
\end{aligned}
$$

The second factor is estimated by Lemma 7.1 if $k_{j+1}-1>k_{j}$ (in which case $B\left(x_{j}\right)\left(k_{j+1}-1-k_{j}\right) \varepsilon \geqq C$, so the condition (128) is satisfied), otherwise it is simply 1 . So

Second factor on the RHS of $(163) \leqq\left\{C\left(1+t B\left(x_{j}\right)\right) e^{-B\left(x_{j}\right)\left(k_{j}+1-1-k_{\jmath}\right) \delta}\right\}^{1-1 / P}$

$$
\leqq C t B \cdot e^{-B\left(x_{j}\right)\left(k_{j+1}-k_{j}\right) \delta}
$$

by (134) and by the definition of $P$.

For the first factor in (163) use that

$$
\left|e^{N_{j}}-e^{\bar{N}_{j}}\right| \leqq\left|e^{N_{j}}-1\right|+\left|e^{\bar{N}_{1}}-1\right|
$$

and recalling the definition of $N_{j}$ (see (114) with $W\left(\tau_{j}\right)=x_{j}$ ) we have

$$
\begin{aligned}
\left|e^{N_{I}}-1\right| \leqq & \frac{1}{2}\left|\int_{\tau_{j}}^{\tau_{j}+1} F^{x_{j}}\left(W(s)-x_{j}\right) \mathrm{d} s\right| \cdot e^{\frac{1}{2} \int_{\tau_{j}}^{\tau_{j}+1} F^{x_{j}}\left(W(s)-x_{j}\right) \mathrm{d} s} \\
& +\left|\int_{\tau_{j}}^{\tau_{j}+1} G^{x_{l}}\left(W(s)-x_{j}\right) \mathrm{d} W(s)\right|+\frac{1}{2}\left|\int_{\tau_{j}}^{\tau_{j}+1} \operatorname{div} G^{x_{J}}\left(W(s)-x_{j}\right) \mathrm{d} s\right|,
\end{aligned}
$$


and similarly for $\bar{N}_{j}$, using the simple estimate $\left|e^{i X+Y}-1\right| \leqq|Y| e^{Y}+|X|$ as before. On the event $E_{j}$, we have $\left|W(s)-x_{j}\right| \leqq 2 R$ and $\left|\bar{W}(s)-x_{j}\right| \leqq 2 R$ for the reflected path $\bar{W}:=T_{j} W$. Therefore (using (134)),

$$
\begin{gathered}
\left|F^{x_{j}}\left(W(s)-x_{j}\right)\right| \leqq 2 c d\left(x_{j}\right) R \leqq C d(x) R, \\
\left|\operatorname{div} G^{x_{j}}\left(W(s)-x_{j}\right)\right| \leqq C d(x) R, \\
\left|G^{x_{j}}\left(W(s)-x_{j}\right)\right| \leqq c d\left(x_{j}\right)\left|W(s)-x_{j}\right|^{2} \leqq C d(x)\left|W(s)-x_{j}\right|^{2},
\end{gathered}
$$

similarly to (125), (135) and (141), and the same estimates are valid for $\bar{W}(s)$ as well. Remark that (169) is valid for any path, while the first two inequalities are valid only for paths in $E_{j}$.

So to estimate the first factor on the right-hand side of (163), we first separate the six different terms obtained in (165) and (166); using the Minkowski inequality then we treat each of them separately. The $\left|\frac{1}{2} \int F^{x_{j}} \mathrm{~d} s\right|^{P} \exp \left(\frac{P}{2} \int F^{x_{j}} \mathrm{~d} s\right)$ and the $\left|\frac{1}{2} \int \operatorname{div} G^{x} \mathrm{~d} s\right|^{P}$ terms are estimated directly by $(C d(x) t R)^{P} \cdot e^{C d(x) t R P}$ and $(C d(x) R t)^{P}$, respectively $\left(\tau_{j+1}-\tau_{j}\right.$ is roughly overestimated by $\left.2 t\right)$.

For the stochastic integral, we use Lemma 7.2 (here with $\mu=P / 2$, recall that $P$ is even) and (169) to obtain a bound

$$
\begin{aligned}
& \mathbf{E}_{\tau_{j}, x_{j}}^{\tau_{j+1}, x_{j+1}}\left|\int_{\tau_{j}}^{\tau_{j+1}} G^{x_{l}}\left(W(s)-x_{j}\right) \mathrm{d} W(s)\right|^{P} \\
& \leqq(C d(x))^{P} \cdot P^{2 P / 3}\left(\left|x_{j}-x_{j+1}\right|^{P}+\eta_{j}^{P / 2}\right)\left[\mathbf{E}_{0, x_{j}}^{2 \eta_{j}, x_{j+1}} \frac{1}{2 \eta_{j}} \int_{0}^{2 \eta_{j}}\left|W^{*}(s)-x_{j}\right|^{8 P} \mathrm{~d} s\right]^{1 / 4},
\end{aligned}
$$

with $2 \eta_{j}:=\tau_{j+1}-\tau_{j}$ and $W^{*}(s):=W\left(s+\tau_{j}\right)$. Recall that we had to omit $\chi\left(E_{j}\right) \chi\left(T_{j}\right.$ exists) since the martingale technique of Lemma 7.2 is not valid for restricted processes. Using the crudest estimates $\eta_{j} \leqq t,\left|x_{j}-x_{j+1}\right| \leqq 2 R$ and the scaling

$$
W^{*}(s)-x_{j}:=\frac{\left(x_{j+1}-x_{j}\right) s}{2 \eta_{j}}+\sqrt{2 \eta_{j}} \cdot b\left(\frac{s}{2 \eta_{j}}\right),
$$

where $b(u)$ is the standard Brownian loop, we have

$$
\begin{aligned}
\text { LHS. of }(170) & \leqq\left(C d(x) P^{2 / 3}\right)^{P}\left(R^{P}+t^{P / 2}\right)\left(\left|x_{j}-x_{j+1}\right|^{2 P}+t^{P} \mathbf{E}^{1 / 4} \int_{0}^{1}|b(u)|^{8 P} \mathrm{~d} u\right) \\
& \leqq\left(C d(x) P^{2 / 3}\right)^{P}\left(R^{P}+t^{P / 2}\right)\left(R^{2 P}+((\text { const }) t P)^{P}\right),
\end{aligned}
$$

using the moments of the standard Brownian loop as well.

Collecting the estimates for the terms in (166) we have

First factor on the RHS of (163)

$$
\begin{aligned}
& \leqq\left[(C d(x) t R)^{P}\left(e^{C d(x) t R P}+1\right)+\left(C d(x) P^{2 / 3}\right)^{P}\left(R^{P}+t^{P / 2}\right)\left(R^{2 P}+((\text { const }) t P)^{P}\right)\right]^{1 / P} \\
& \leqq C d(x) t^{3 / 2}(B t)^{13 / 6} \leqq \frac{C}{(B t)^{3}},
\end{aligned}
$$


where, in the calculation, we used that $B t \geqq 1$, the explicit value of $R=8 t \sqrt{B}$ and $P=2\left[t B\left(x_{j}\right)\right]+2 \leqq C B t$ (by (134)). The last line of (173) shows that the critical exponent in the definition of $d(x)$ (see (12)), determining the maximal growth rate of $B(x)$ at infinity, must be at least $31 / 6$ in order to obtain the $C(B t)^{-3}$ estimate which is necessary for the rest of the proof. This completes the proof of Lemma 7.3.

Finally, after estimating the last three lines of (157) by quantities independent of $x_{j}$ 's (see (162), (163), (164) and (173)), we can drop the condition $\left|x-x_{j}\right| \leqq R$ on the range of integration and use the semigroup property of the heat kernel to perform the $x_{j}$ integrations. Therefore

$$
\text { LHS of }(150) \leqq \sum_{n=1}^{2 t / \varepsilon} \frac{1}{2^{n}} \sum_{0<k_{1}<\cdots<k_{n} \leqq 2 t / \varepsilon}\left(\frac{C}{(B t)^{2}}\right)^{n} \cdot C B t .
$$

Finally, use that $k_{n} \leqq 2 t / \varepsilon=[B t]+1$, therefore the sum over all possible $0<k_{1}<\cdot<k_{n}$ contains altogether $\left(\begin{array}{c}{[B t]+1} \\ n\end{array}\right)$ choices. So eventually, we have

$$
\begin{aligned}
\text { LHS of }(150) & \leqq \sum_{n=1}^{[B t]+1} \frac{1}{2^{n}}\left(\begin{array}{c}
{[B t]+1} \\
n
\end{array}\right)\left(\frac{C}{(B t)^{2}}\right)^{n} \cdot C B t \\
& \leqq\left[\left(1+\frac{C}{2(B t)^{2}}\right)^{[B t]+1}-1\right] \cdot C B t \leqq C
\end{aligned}
$$

using $B t \geqq 1,[B t]+1 \leqq 2 B t$ and the fact that the function

$$
X \rightarrow\left[\left(1+\frac{C}{X^{2}}\right)^{X}-1\right] \cdot X
$$

is bounded uniformly for $X \geqq 1$ by a constant depending only on $C$ (use it for $X:=2 B t$ ). The estimate (175) finishes the proof of Main Lemma 6.2 and Theorem 2.2.

\section{Appendices}

\section{A. Selfadjointness and Negative Essential Spectrum}

Here we clarify some point about the selfadjointness and the negative essential spectrum of $\mathbf{H}_{\text {Pauli }}$, and we show that the various Birman-Schwinger kernels used in the proofs are compact.

Let us start with the Birman-Schwinger kernels (see (25)-(27) and (46)-(48)), which are apriori defined on $C_{0}^{\infty}$ as $|V+E / 2|_{-} \in L^{1}$ for any $E>0$. Assuming that the right-hand side of each Lieb-Thirring inequality (see (4), (7) and (18)) is finite, we have shown, in particular, that $\operatorname{Tr}\left(K_{E, L}^{<}\right), \operatorname{Tr}\left[\left(K_{E, L}^{>}\right)^{2}\right]$ and $\left.\operatorname{Tr}\left(\mathbf{K}_{E, L}^{>}\right)^{2}\right]$ are finite (since these quantities are monotone decreasing functions of $E$, and their integrals were shown to be finite, see (35) and (40)), therefore $K_{E, L}^{<}, K_{E, L}^{>}$and $\mathbf{K}_{E, L}^{>}$ are compact. It is easily seen from (49) that for $\varrho>0$,

$$
\#\left\{\text { ev's of } \mathbf{K}_{E, L}^{<} \text {bigger than } \varrho\right\} \leqq(\text { const }) \cdot \varrho^{-\gamma} \int|V|_{-}^{\gamma}<\infty
$$


(where the constant depend on $L,\|\mathbf{B}\|$ and $\gamma$ ), in particular, $\mathbf{K}_{E, L}^{<}$is also compact. Therefore the Birman-Schwinger kernels $K_{E}$ and $\mathbf{K}_{E}$ are compact operators. This information has been used in (28) and in (49). Moreover, this shows that $\mathrm{V}+$ $E / 2 L_{-}$is a relatively compact perturbation of $H+p_{3}^{2}+E / 2$ and of $\mathbf{H}_{\text {Pauli }}^{0}+E / 2$. Therefore

$$
\sigma_{\text {ess }}\left(\mathbf{U}_{E}\right)=\sigma_{\text {ess }}\left(\mathbf{H}_{\text {Pauli }}^{0}+\frac{E}{2}\right) \subset\left[\frac{E}{2}, \infty\right]
$$

with $\mathbf{U}_{E}:=\mathbf{H}_{\text {Pauli }}^{0}+E / 2-|V+E / 2|$, which is bounded from below being a relatively compact perturbation of a positive operator. But

$$
\mathbf{H}_{\text {Paul }}^{0}=\mathbf{U}_{E}+\left|V+\frac{E}{2}\right|_{+}-E \geqq \mathbf{U}_{E}-E,
$$

so, in particular, $\mathbf{H}_{\text {Paul }}$ is bounded from below, and therefore it has a selfadjoint extension. Furthermore, using that if two operators $X \leqq Y$ are bounded from below, then inf $\sigma_{e s s}(X) \leqq \inf \sigma_{e s s}(Y)$, we have

$$
\inf \sigma_{e s s}\left(\mathbf{H}_{\text {Pauli }}\right) \geqq \inf \sigma_{e s s}\left(\mathbf{U}_{E}-E\right) \geqq-\frac{E}{2}
$$

by (179). Since this is true for any $E>0$, we obtain that $\mathbf{H}_{\text {Pauli }}$ has no negative essential spectrum. The same statement for $H_{0}$ is proved similarly.

\section{B. Counterexample}

For any $\gamma \geqq 0$ and given constants $C_{1}$ and $C_{2}$ we construct a special magnetic field $B(x)$ and potential $V(\mathbf{x})$ such that the $\gamma^{\text {th }}$ moment of the negative eigenvalues of $H_{0}$ is not bounded by

$$
C_{1} \int_{\mathbf{R}^{3}} B(x)|V(\mathbf{x})|_{-}^{\gamma+1 / 2} \mathrm{~d} \mathbf{x}+C_{2} \int_{\mathbf{R}^{3}}|V(\mathbf{x})|_{-}^{\gamma+3 / 2} \mathrm{~d} \mathbf{x} .
$$

The key idea is that we will choose $B$ and $V$ such that their supports be disjoint, so the first term disappears in the possible bound (181). Then we will show that the sum of the negative eigenvalues behaves at least like (const) $N$ if we rescale the magnetic field by $N^{2}$, but this rescaling clearly does not effect the bound (181).

For the proof, choose a one-dimensional potential $v$ with $|v|-\in L^{5 / 2}(\mathbf{R})$ such that $p_{3}^{2}+v\left(x_{3}\right)$ has a negative eigenvalue $-\lambda$, and let $\psi\left(x_{3}\right)$ be the corresponding normalized eigenfunction. (E.g. $v\left(x_{3}\right)=x_{3}^{2}-2,-\lambda=-1, \psi\left(x_{3}\right)=\pi^{-1 / 4} e^{-x_{3}^{2} / 2}$.) Let $V(\mathbf{x}):=v\left(x_{3}\right) \chi(|x| \leqq 1)$, i.e. the potential is supported in a cylinder built over the unit disc in $\mathbf{R}^{2}$; and let $B(x)=N^{2} \chi(|x| \geqq 1)$ where $N$ is a free positive parameter, $B:=N^{2}$. In this case the conjecture (4) says that

$$
\sum_{i}\left|E_{l}\right|^{\gamma} \leqq C_{2} \pi \int_{\mathbf{R}}\left|v\left(x_{3}\right)\right|_{-}^{\gamma+3 / 2} d x_{3}
$$

independently of $B=N^{2}$. On the other hand, we will show that $\sum_{i}\left|E_{i}\right|^{\gamma} \geqq$ (const) $\cdot N$. Notice that $B(x)$ is not continuous since the calculation happens to be simpler in this way, but the same idea easily provides a counterexample with a $C^{\infty}$ magnetic field which is sufficiently close to $B(x)$. 
We will use the complex notation $z:=x_{1}+i x_{2}$ for $x=\left(x_{1}, x_{2}\right)$ in the plane of the first two coordinates. As it is explained in the proof of the AharonovCasher theorem (see [AC, CFKS, Sect. 6.4]), the ground state eigenfunctions of $H=(p-A(x))^{2}-B(x)$ can be found in the form of $e^{h(z)} g(z)$, where $h$ satisfies $-\Delta h(z)=B(z)$ and $g(z)$ is analytic.

In our case let $h(z)$ be the following function:

$$
h(z):= \begin{cases}-B / 4 & \text { for }|z| \leqq 1 \\ -B\left(\log |z|^{2}-|z|^{2}\right) / 4 & \text { for }|z|>1\end{cases}
$$

then clearly $-\Delta h(z)=B(z)$ for the $B(z)=B(x)$ defined above. The functions

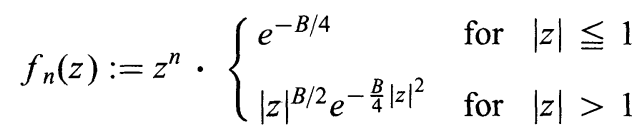

$(n=0,1,2, \ldots)$ are ground states of $H$, and they are orthogonal in $L^{2}(\mathbf{C})=L^{2}\left(\mathbf{R}^{2}\right)$. Define $F_{n}(\mathbf{x}):=f_{n}(x) \psi\left(x_{3}\right)$, then $\left\|F_{n}\right\|_{L^{2}\left(\mathbf{R}^{3}\right)}=\left\|f_{n}\right\|_{L^{2}\left(\mathbf{R}^{2}\right)}$, and they are all linearly independent since they are in different angular momentum sectors. By the variational principle (applied separately to each sector)

$$
\sum_{i}\left|E_{i}\right|^{\gamma} \geqq \sum_{n=0}^{K_{N}-1}\left|\frac{\left(F_{n}, H_{0} F_{n}\right)}{\left\|F_{n}\right\|^{2}}\right|^{\gamma},
$$

where $K_{N}$ is any integer (to be determined later) not greater than the number of negative eigenvalues (with multiplicity). Computing

$$
\left(F_{n}, H_{0} F_{n}\right)=\|\nabla \psi\|_{L^{2}(\mathbf{R})}^{2} \int_{|x| \geqq 1}\left|f_{n}(x)\right|^{2} \mathrm{~d} x-\lambda \cdot \int_{|x|<1}\left|f_{n}(x)\right|^{2} \mathrm{~d} x
$$

(using $H_{0}=H+p_{3}^{2}+V$ and $H f_{n}=0$ ), we have that

$$
\sum_{i}\left|E_{i}\right|^{\gamma} \geqq \sum_{n=0}^{K_{N}-1}\left|\frac{\lambda-T_{n}\|\nabla \psi\|_{L^{2}(\mathbf{R})}^{2}}{T_{n}+1}\right|^{\gamma},
$$

where

$$
T_{n}:=\frac{\left\|f_{n}\right\|_{\text {out }}^{2}}{\left\|f_{n}\right\|_{\text {in }}^{2}}:=\frac{\int_{|x| \geqq 1}\left|f_{n}(x)\right|^{2} \mathrm{~d} x}{\int_{|x|<1}\left|f_{n}(x)\right|^{2} \mathrm{~d} x}
$$

is the ratio of the norms of $f_{n}$ outside and inside the unit disc.

The inside norm is easily computed: $\left\|f_{n}\right\|_{i n}^{2}=\frac{\pi}{n+1} e^{-B / 2}$. The outside norm can be estimated from above as follows (in polar coordinates):

$$
\begin{aligned}
\left\|f_{n}\right\|_{\text {out }}^{2} & =2 \pi \int_{1}^{\infty} r^{2 n+B+1} e^{-\frac{B}{2} r^{2}} \mathrm{~d} \tau \leqq 2 \pi \int_{0}^{\infty} r^{2 n+B+1} e^{-\frac{B}{2} r^{2}} \mathrm{~d} r \\
& =\frac{2 \pi}{B}\left(\frac{2}{B}\right)^{n+B / 2} \int_{0}^{\infty} t^{n+B / 2} e^{-t} \mathrm{~d} t
\end{aligned}
$$


The gamma integral is estimated by the Stirling formula, yielding

$$
\left\|f_{n}\right\|_{\text {out }}^{2} \leqq \frac{\text { const }}{\sqrt{B}} e^{-B / 2}
$$

for $n \leqq(e-2) B$, so $T_{n} \leqq c_{0} \frac{n+1}{\sqrt{B}}=c_{0} \frac{n+1}{N}$ with some universal $c_{0}>0$.

Choose

$$
K_{N}:=\left[\frac{N \lambda}{2 c_{0}\|\nabla \psi\|_{L^{2}(\mathbf{R})}^{2}}\right]
$$

([x] denotes the integer part), then by (186) there are at least $K_{N}$ negative eigenvalues, since $\left(F_{n}, H_{0} F_{n}\right)<0$ for $0 \leqq n \leqq K_{N}-1$ (and $n \leqq(e-2) B$ is also satisfied). So by (187) and (191)

$$
\sum_{i}\left|E_{i}\right|^{\gamma} \geqq \sum_{n=0}^{K_{N}-1}\left(\frac{\lambda N-c_{0}(n+1)\|\nabla \psi\|_{L^{2}(\mathbf{R})}^{2}}{c_{0}(n+1)+N}\right)^{\gamma} \geqq(\text { const }) \cdot N,
$$

where this last positive constant depends on everything except $N$.

Acknowledgements. The author wishes to express his gratitude to Elliott H. Lieb, Michael Aizenman and Jan Philip Solovej for their continuous help and encouragement, and to Princeton University for its financial support during his graduate studies. Part of this work has been done in the stimulating environment of the Erwin Schrödinger Institute in Vienna.

\section{References}

[AC] Aharonov, Y., Casher, A.: Ground state of spin-1/2 charged particle in a twodimensional magnetic field. Phys. Rev. A19, 2461-2462 (1979)

[AHS] Avron, J., Herbst, I., Simon, B.: Schrödinger operators with magnetic fields. I. General interactions. Duke Math. J. 45, 847-883 (1978)

[BHL] Broderix, K., Hundertmark, D., Leschke, H.: Continuity properties of Schrödinger semigroups with magnetic fields. In preparation

[C] Carmona, R.: Regularity properties of Schrödinger and Dirichlet semigroups. J. Funct. Anal. 33, 259-296 (1979)

[CFKS] Cycon, H.L., Froese, R.G., Kirsch, W., Simon, B.: Schrödinger Operators with Application to Quantum Mechanics and Global Geometry. Berlin-Heidelberg-New York: Springer-Verlag, 1987

[CdV] Colin de Verdiére, Y.: L'asymptotique de Weyl pour les bouteilles magnétiques. Commun. Math. Phys. 105, 327-335 (1986)

[DJS] De Angelis, G.F., Jona-Lasinio, G., Sirugue, M.: Probabilistic solution of Pauli type equations. J. Phys. A: Math. Gen. 16, 2433-2444 (1983)

[E-1993] Erdős, L.: Ground state density of the two-dimensional Pauli operator in the strong magnetic field. Lett. Math. Phys. 29, 219-240 (1993)

[E-1994(a)] Erdös, L.: Estimates on stochastic oscillatory integrals and on the heat kernel of the magnetic Schrödinger operator. Duke Math. J. 76, 541-566 (1994)

[E-1994(b)] Erdös, L.: Magnetic Lieb-Thirring inequalities and stochastic oscillatory integrals. $\mathrm{Ph}$. D. Thesis, Princeton University, 1994

[FLL] Fröhlich, J., Lieb, E.H., Loss, M.: Stability of Coulomb systems with magnetic fields. Commun. Math. Phys. 104, 251-270 (1986)

[GS] Gihman, I.I., Skorohod, A.V.: Stochastic Differential Equations. Berlin: Springer, 1972

[K] Kato, T.: Perturbation Theory for Linear Operators. Berlin-Heidelberg-New York: Springer Verlag, 1966 
[L] Lieb, E.H.: The number of bound states of one-body Schrödinger operators and the Weyl problem. In: Proceedings of Symposia in Pure Mathematics. Volume 36, 1980, pp. $241-251$

[LT] Lieb, E.H., Thirring, W.: Inequalities for the moments of the eigenvalues of the Schrödinger Hamiltonian and their relation to Sobolev inequalities. In: Studies in Mathematical Physics, Essays in Honor of Valentine Bargmann. Eds. Lieb, E.H., Simon, B., Wightman, A.S., Princeton, NJ: Princeton Univ. Press, 1976, pp. 269-304

[LSY-I] Lieb, E.H., Solovej, J.P., Yngvason, J.: Asymptotics of heavy atoms in high magnetic fields: I. Lowest Landau band regions. To appear in Commun. Pure Appl. Math.

[LSY-II] Lieb, E.H., Solovej, J.P., Yngvason, J.: Asymptotics of heavy atoms in high magnetic fields: II. Semiclassical regions. Commun. Math. Phys. 161, 77-124 (1994)

[LSY-III] Lieb, E.H., Solovej, J.P., Yngvason, J.: In preparation. An announcement of the result was made in Quantum dots. To appear in the Proceedings of the International Conference on Partial Differential Equations and Mathematical Physics, held at the University of Alabama, Birmingham, March 1994

[M-1990] Matsumoto, H.: The short time asymptotics of the traces of the heat kernels for the magnetic Schrödinger operators. J. Math. Soc. Japan 42, 677-689 (1990)

[M-1991] Matsumoto, H.: Classical and non-classical eigenvalue asymptotits for the magnetic Schrödinger operators. J. Funct. Anal. 95, 460-482 (1991)

[MS] Miller, K., Simon, B.: Quantum magnetic Hamiltonians with remarkable spectral properties. Phys. Rev. Lett. 44, 1706-1707 (1980)

[RS] Reed, M., Simon, B.: Methods of Modern Mathematical Physics, I-IV. New York: Academic Press, 1972-79

[S-1979] Simon, B.: Functional Integration and Quantum Physics. New York: Academic Press, 1979

[S-1979(a)] Simon, B.: Maximal and minimal Schrödinger forms. J. Operator Theory 1, 37-47 (1979)

[S-1982] Simon, B.: Schrödinger semigroups. Bull. Am. Math. Soc. 7, 447-526 (1982)

[S-1984] Simon, B.: Semiclassical analysis of low lying eigenvalues. II. Tunneling. Ann. Math. 120, 89-118 (1984)

[Sob] Sobolev, A.: The quasiclassical asymptotics of local Riesz means for the Schrödinger operator in a strong homogeneous magnetic field. Duke Math. J. 74, 319-429 (1994)

[T] Tamura, H.: Asymptotic distribution of eigenvalues for Schrödinger operators with homogeneous magnetic fields. Osaka J. Math. 25, 633-647 (1988)

[Y] Yor, M.: Some aspects of the Brownian motion. Prépublication No. 104 du Laboratoire de Probabilités de l'Université Paris VI, 1992 